\title{
Interpreting Ideality Factors for Planar Perovskite Solar Cells: Ectypal Diode Theory for Steady-State Operation
}

\author{
N.E. Courtier $\circledast^{*}$ \\ Mathematical Sciences, University of Southampton, Southampton, United Kingdom
}

(Received 10 March 2020; revised 20 May 2020; accepted 10 July 2020; published 12 August 2020)

\begin{abstract}
Ideality factors are used to identify the dominant form of recombination in many types of solar cells and guide future development. Unusual noninteger and voltage-dependent ideality factors, which are difficult to explain using the classical diode theory, have been reported for perovskite solar cells and remain unexplained. Experimental measurements and theoretical simulations of the electric potential profile across a planar perovskite solar cell show that significant potential drops occur across each of the perovskiteand transport-layer interfaces. Such potential profiles are fundamentally distinct from the single potential drop that characterizes a $p-n$ or a $p-i-n$ junction. We propose an analytical model, developed specifically for perovskite devices, in which the ideality factor is replaced by a systematically derived analog, which we term the ectypal factor. In common with the classical theory, the ectypal diode equation is derived as an approximation to a drift-diffusion model for the motion of charges across a solar cell, however, crucially, it incorporates the effects of ion migration within the perovskite absorber layer. The theory provides a framework for analyzing the steady-state performance of a perovskite solar cell (PSC) according to the value of the ectypal factor. Predictions are verified against numerical simulations of a full set of drift-diffusion equations. An important conclusion is that our ability to evaluate PSC performance, using standard techniques such as the analysis of dark $J-V$ or Suns- $V_{\mathrm{OC}}$ measurements, relies on understanding how the potential distribution varies with applied voltage. Implications of this work on the interpretation of data from the literature are discussed.
\end{abstract}

DOI: 10.1103/PhysRevApplied.14.024031

\section{INTRODUCTION}

The Shockley diode equation is commonly employed in solar-cell research to diagnose the recombination mechanism that limits the performance of a particular device architecture [1]. The diode model is derived from the theory of drift and diffusion of charge carriers across a solar cell, including the generation and recombination of charge carrier pairs (electrons and holes). Though approximate, this model provides a useful description of the currentvoltage $(J-V)$ characteristics of many types of solar cell. The value of the diode ideality factor is key for the diagnosis of both the type of recombination that limits cell performance and its location within the device. It is therefore crucial to be able to correctly evaluate and interpret the value of the ideality factor. However, in the case of metal halide perovskite solar cells (PSCs), this approach has led

*n.e.courtier@soton.ac.uk

Published by the American Physical Society under the terms of the Creative Commons Attribution 4.0 International license. Further distribution of this work must maintain attribution to the author(s) and the published article's title, journal citation, and DOI. to some unexpected results, which cast doubt on its validity in assessing this promising photovoltaic technology [2-6]. Perovskite solar cells have recorded a rapid rise in power conversion efficiency over a short but intense period of research effort $[7,8]$. However, the cells currently lack long-term stability [9]. Success in the economic market (most likely via their incorporation into tandem devices with silicon $[10,11]$ ) will depend on our understanding of the fundamental physical mechanisms that control the behavior of PSCs.

Experimental studies have reported ideality factors for PSCs ranging from 0.9 [12] to higher than 5 [2]. Such values lie outside the typical range of values (1-2) predicted and explained by the theory behind the classical Shockley diode equation. Many noninteger values, for example, between 1 and 2, have also been reported [13-17], but are difficult to explain using the classical theory. A summary of common and uncommon interpretations is given in Ref. [3]. In the field of dye solar cells, noninteger ideality factors have been explained by the existence of a broad distribution of trap states combined with trap-limited recombination $[18,19]$. Noninteger ideality factors, including values smaller than 1 , can also occur as a result of energetic misalignment between the absorber and contact materials [20-22]. 
In both of the experimental studies mentioned above $[2,12]$, the values of the ideality factor are calculated from measurements of the open-circuit voltage $\left(V_{\mathrm{OC}}\right)$ of a PSC under different illumination intensities. This is often referred to as the Suns- $V_{\mathrm{OC}}$ method. Interestingly, Pockett et al. [2] report that the ideality factors for a set of 12 planar PSCs, prepared at the same time, cluster around two distinct values (approximately 2.6 and 5.2). Tress et al. [6] use three different experimental techniques to calculate the ideality factor for four types of PSC. The value of the ideality factor is shown to vary substantially depending on how it is measured. Values calculated from the derivative of a $J-V$ curve measured in the dark, referred to as the dark $J-V$ method, are shown to strongly depend on the applied voltage. Tress et al. [6] conclude that the Suns$V_{\mathrm{OC}}$ method provides the most reliable values, while the ambiguity in the dark $J-V$ results is attributed to voltagedependent parasitic resistances, although the exact origins are not known. Contreras-Bernal et al. [15,23] use an alternative method in which the ideality factor is calculated from the high-frequency impedance response of a PSC measured at open-circuit under different illumination intensities. Voltage-dependent ideality factors are also published in Ref. [24,25] and the Supplemental Material of Ref. [3]. These recent findings highlight the need for a clear picture of the principle working mechanism of a PSC, which can be used to interpret and explain the unusual ideality factors of PSCs. This picture can be obtained from a detailed drift-diffusion model for a PSC based on the microscopic properties of the device.

Combined experimental and theoretical studies, which seek to understand the performance limitations of state-ofthe-art PSCs, such as Refs. [3,4,16], conclude that current techniques for analysis do not provide reliable conclusions. Almora et al. [3] and Calado et al. [4] attribute the difficulty in determining a unique value for the ideality factor to the interference of undesirable hysteresis often observed in $J-V$ measurements [26-29]. The origin of this $J-V$ hysteresis is most commonly attributed to the migration of mobile ionic charge within the perovskite material. Recent reviews that discuss the impact of ion migration on the future prospects of PSCs are given in Ref. [30,31]. The presence of mobile ionic charge in PSCs means that use of the classical diode theory to analyze their behavior is not physically justified and therefore traditional interpretations of the ideality factor can be misleading.

Using the hypothesis that ion migration is the origin of $J-V$ hysteresis, Calado et al. [4] explain that it is the evolving profile of the electric potential across a PSC that hampers the measurement of a cell's $V_{\mathrm{OC}}$, and thus the ideality factor, by conventional means. It is noted that the interpretation of a steady-state ideality factor should take into account the homogeneity of charge-carrier densities across the perovskite layer. An alternative method for the determination and interpretation of a "transient ideality factor" is suggested, that involves comparing timedependent measurements to the results of drift-diffusion simulations. However, the suggested method depends on preconditioning the cell in a particular initial state and determining the cell's characteristics from a transient measurement, rather than from the steady-state conditions at which a cell is expected to operate.

Drift-diffusion models incorporating mobile ionic charge have been shown to be capable of reproducing a wide variety of experimentally observed behavior [32-35]. In PSCs, a high density of mobile ionic charge is predicted to exist within the perovskite material [36]. As a result, the distribution of the electric field is controlled by the position of the mobile ions and evolves on a slower timescale than that of the charge-carrier dynamics [4,33,37]. At steady state, ionic accumulation or depletion at the edges of the perovskite absorber layer (PAL) screens the electric field from the bulk of the PAL and causes potential drops to form across the interfaces between the PAL and each of the transport layers (TLs). As such, the existence of mobile ionic charge in a PSC produces a notably different potential profile from that of either a $p-n$ or $p-i-n$ junction, as shown in Fig. 1. The electric potential across the three core layers of a PSC displays a linear profile across the bulk of each layer and rapid changes across narrow regions, called Debye layers, immediately either side of each interface. Such profiles have been measured experimentally using Kelvin probe force microscopy (KPFM) and presented in Ref. [38-40]. The proportion of the total potential difference that falls across each Debye layer depends on the relative values of the permittivity and the mean density of the majority mobile charge species of each material layer [33].

The formation of potential drops due to charge accumulation at the absorber interfaces has also been found to be significant in the study of thin-film silicon solar cells [41]; although in such cells, the charge accumulation is of an electronic nature and only screens a small proportion of the total potential drop. The model for a $p-i-n$ solar cell presented by Taretto [41] predicts voltage-dependent ideality factors due to charge accumulation within the intrinsic absorber layer and shows improved agreement with experimental data compared to classical diode theory. As such, Taretto [41] demonstrates how analytical modeling can be used as a practical tool to elucidate the effects of key material properties on the performance of a solar cell.

In this work, we develop a simple analytical model appropriate for planar PSCs, which we term the ectypal [42] diode theory. This model describes the principle working mechanism of a planar PSC operating in steady-state conditions and can be used to quantitatively assess cell performance in an analogous way to the classical diode theory. The proposed model is systematically derived from a drift-diffusion model for ion migration and charge transport across a PSC and validated against numerical solution 
of the full drift-diffusion model. In particular, we derive a modified relationship between the current density $(J)$ and the applied voltage $(V)$ for steady-state measurements, that incorporates the effects of ionic charge accumulation at the interfaces, and use it to explain the occurrence of anomalous ideality factors in the literature. In this model, recombination losses are controlled by the electric potential distribution, which is dependent on the distribution of the ions within the PAL.

Crucially, the ectypal diode model is able to explain the unusual noninteger and voltage-dependent values of the "ideality factor" reported for PSCs without having to resort to nonstandard theories of charge-carrier recombination. Similar to the classical diode equation, the derived expression for $J(V)$ separates into a generation term (which depends only on the light intensity and absorption properties of the device) subtracted by an expression for the dark current density (which depends only on the losses due to recombination). Unlike the classical theory, the proposed model is tailored to the distribution of the electric field that characterizes a planar PSC (see Fig. 1). In contrast to Refs. $[4,41]$, we find that, for PSCs, the voltage dependence of the "ideality factor" does not originate from the spatial distribution of electronic charge carriers within the PAL. Instead we show that the voltage dependence is an expected result of the nonlinear capacitance relations that describe the accumulation of charge in the Debye layers that exist either side of each PAL-TL interface [33,37]. We find that measurements of the "ideality factor" are voltage dependent even when measured under steady-state conditions.

In Sec. II, we outline the classical theory for a solar cell and two standard methods for analyzing its performancelimiting recombination mechanism, namely the dark $J-V$ method and the Suns- $V_{\mathrm{OC}}$ method. We then, in Sec. III, describe the proposed ectypal diode theory that is tailored to the physical properties of a PSC. We also give details of the numerical simulations used to verify the model. In Sec. IV, we analyze, using the two standard methods, two example sets of cell parameter values for five separate cases, in which the cell is limited by one of five different recombination mechanisms. The example cells are simulated using the open-source IonMonger simulation tool [43] and the results are explained using the ectypal diode theory. Lastly, in Sec. V, we discuss the implications of this work on experimental results from the literature before drawing our conclusions. Thus, we show that the proposed model provides a coherent framework for analyzing a PSC and represents a significant step forward in our understanding of the steady-state characteristics of these devices.

\section{BACKGROUND}

The classical Shockley diode equation is derived from drift-diffusion theory and can be used to analyze the $J-V$ characteristics of a solar cell with a $p-n$ or $p-i-n$ architecture [1]. The Shockley diode equation is derived in the small current limit $[44,45]$ and holds only where the charge-carrier generation and the current extracted from the cell are both sufficiently small that the charge-carrier distributions are in approximate quasiequilibrium, and therefore close to being Boltzmann distributed. For relatively efficient photovoltaic cells, the range of validity of this approximation extends into the operating regime of the cell (at 1 Sun) and from short circuit to open circuit, and so can be used to fit experimental $J-V$ curves. As outlined below, the identification of the ideality factor $n_{\text {id }}$ from the fit to the diode equation is frequently used to infer information about the dominant recombination in the cell.

The ideal diode equation (with ideality factor 1) describes a cell limited by bimolecular band-to-band recombination occurring within the absorber layer, while the nonideal diode equation includes an ideality factor,

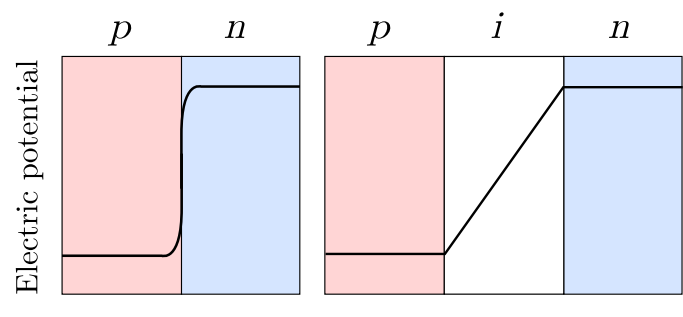

Ideal and nonideal diode equation

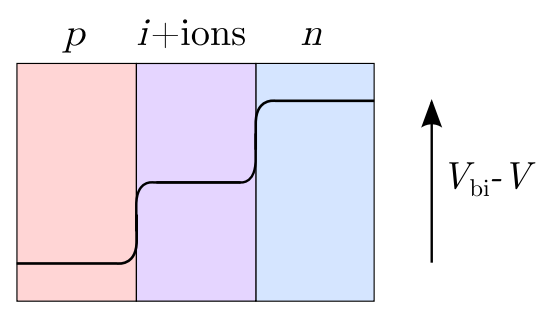

Ectypal diode equation

FIG. 1. Sketch showing the steady-state distributions of the electric potential across three solar-cell architectures, when held at short circuit. The architectures are those of a $p-n$ junction, a $p-i-n$ junction and a planar PSC architecture in which at least one species of mobile ionic charge exists in the perovskite absorber layer. The classical (ideal or nonideal) diode equation is valid for both the $p$ - $n$ and $p-i-n$ solar-cell architectures, however, it is not valid for a PSC held at steady state due to the effect of the ionic charge on the distribution of the electric potential across the cell. An analogous equation for a PSC (such as the ectypal diode equation proposed in this paper) must take into account the form of the potential around the two perovskite- and transport-layer interfaces that occurs due to the accumulation or depletion of ionic charge. 
which extends the model to take into account losses via different recombination pathways. The nonideal diode equation is given by

$$
\begin{gathered}
J(V)=J_{s}-J_{\text {dark }}(V), \\
J_{\text {dark }}(V)=J_{0}\left[\exp \left(\frac{V}{n_{\mathrm{id}} V_{T}}\right)-1\right],
\end{gathered}
$$

in which $J_{s}$ is the photogenerated current density (inside the cell), $J_{\text {dark }}$ is the dark current density (due to recombination), $J_{0}$ is the saturation current density, $n_{\text {id }}$ is the ideality factor, and $V_{T}=k_{B} T / q$ is the thermal voltage. Here, $k_{B}$ is the Boltzmann constant, $T$ is the temperature, and $q$ is the elementary charge. In the ideal case of direct radiative recombination, $n_{\mathrm{id}}=1$. When other types of bulk recombination dominate, the ideality factor is expected to equal $n_{\text {id }}=2 / \gamma$ where $\gamma$ is the reaction order, i.e., $n_{\text {id }}=$ 2 indicates first-order bulk recombination, $n_{\mathrm{id}}=1$ indicates bimolecular bulk recombination and $n_{\mathrm{id}}=\frac{2}{3}$ signifies trimolecular Auger recombination [1,20,46]. A value of $n_{\text {id }}=2$ is typically interpreted as trap-assisted ShockleyRead-Hall (SRH) recombination. Meanwhile, $n_{\text {id }}=1$ can also be the result of dominant surface recombination. Other, less common, interpretations are summarized in the Supplemental Material of Ref. [3], such as recombination due to a nonuniform distribution of recombination centres. Calado et al. [4] provide multiple interpretations of ideality factors equal to 1 or 2 , which are due to either SRH or band-to-band recombination mechanisms.

In reality, the value of an ideality factor can vary with voltage due to a change in the dominant form of recombination. This scenario can be described using a two-diode model. However, the associated increase in the number of fitting parameters means that this approach can yield erroneous results. This approach is also not able to explain the unusual values of the ideality factor that have been reported for PSCs in the literature, in particular, those outside of the range 1-2 and those that vary slowly with applied voltage. The classical model can also be extended by incorporating the effects of external series and shunt and parallel resistance into the classical diode equation [1]. However, fitting series and shunt resistance parameters to experimental data provides little information about the location of recombination losses and also does not seem to explain the anomalous voltage-dependent values of the ideality factor reported for PSCs. So, in this work, we assume that just one recombination mechanism controls the observed behavior and that the losses due to external resistances are negligible for a high-efficiency cell operating in its power-generating regime.

The value of the ideality factor $n_{\text {id }}$ can be estimated using a number of different experimental procedures. Tress et al. [6] describe how $n_{\text {id }}$ may be calculated from fitting (a) measurements of dark $J$ - $V$ curves, (b) open-circuit voltage versus illumination intensity measurements (known as the Suns- $V_{\mathrm{OC}}$ method), and (c) electroluminescence measurements. The dark $J-V$ method is also used in Refs. $[24,25,40]$, while the Suns- $V_{\mathrm{OC}}$ method is also used in Refs. $[2,4,12,16,34]$. We consider both the dark $J-V$ and Suns- $V_{\mathrm{OC}}$ methods in this work.

For positive applied voltages $V \gg V_{T}$ (usually taken to mean $V \gtrsim 0.1 \mathrm{~V}$ ), the exponential term in the dark current density in Eq. (1b) dominates so that the Shockley diode equation can be approximated by

$$
J(V)=J_{s}-J_{0} \exp \left(\frac{V}{n_{\mathrm{id}} V_{T}}\right) .
$$

In the dark $J-V$ method, the photogenerated current density $J_{s}$ is zero and so the ideality factor is calculated from the slope of the logarithm of the dark current density from Eq. (2) versus applied voltage, via

$$
n_{\text {id }}=\frac{1}{V_{T}}\left(\frac{\partial \ln J_{\text {dark }}}{\partial V}\right)^{-1} .
$$

Though the value of an ideality factor is expected to be approximately constant, Wetzelaer et al. [24] show, using this method, that the value of the ideality factor for a planar PSC is strongly voltage dependent and varies with temperature. Other reports of strongly voltage-dependent ideality factors for a variety of PSC architectures are given in Refs. $[6,24,25]$.

The Suns- $V_{\mathrm{OC}}$ method relies on the assumption that the photogenerated current density $J_{S}$ is proportional to the illumination intensity and the dominant recombination mechanism does not vary with illumination. In this case, measurements of the open-circuit voltage over a range of illumination intensities can be used to determine the ideality factor via

$$
n_{\mathrm{id}}=\frac{1}{V_{T}} \frac{\partial V_{\mathrm{OC}}}{\partial \ln F_{\mathrm{ph}}},
$$

where $F_{\mathrm{ph}}$ is the incident photon-flux density. The Suns$V_{\mathrm{OC}}$ method is deemed to be more reliable than the dark $J$ $V$ method because measurements performed at open circuit are not affected by parasitic series resistance.

\section{METHODS}

\section{A. Surface-polarization model}

The fundamental difference between the classical model and the surface-polarization model for a PSC lies in the distribution of the electric field across a device, see Fig. 1. Both models are derived from drift-diffusion theory for the transport of mobile charges in one dimension across a device. In the classical model for a $p-n$ or $p-i-n$ junction, 
there is one significant potential drop (or diode junction) within the device, which acts to separate the photogenerated charge. However, across a planar PSC two major potential drops have been observed to exist, one at each of the interfaces between the PAL and one of the transport layers [38-40], as depicted in Fig. 2. Such a distribution is also predicted by drift-diffusion simulations in which the electric field across a PSC depends on the distribution of a large density of mobile ionic charge, as well as the electronic charge in the device. The distribution of the electric potential is crucial for determining the performance of a cell because current losses (due to recombination occurring either within the bulk or at the interfaces) depend on both the potential energy that must be overcome for charge carriers to recombine as well as the local charge-carrier concentrations, which in turn also depend on the potential. Therefore, current losses strongly depend on the location of potential drops within a cell.

Figure 2 shows the typical form of a steady-state potential profile across the three core layers of a planar PSC. The total potential drop across the cell is entirely taken up within four narrow Debye layers either side of the PALTL interfaces. These potential drops are labeled $V_{1-4}$. The potential is expected to take this form in a PSC that satisfies the following assumptions of the surface-polarization model [33] that: (i) a high density of mobile ionic charge exists within the PAL, in comparison to the densities of electronic charge carriers that exist under illumination intensities up to the equivalent of 1 Sun, (ii) the Debye length associated with the majority species of mobile charge in each material layer is much smaller than the width of that layer, and (iii) the timescale for equilibration of the charge carriers in the TLs is much faster than that of the mobile ionic charge in the perovskite. For a mean ion vacancy density of $1.6 \times 10^{25} \mathrm{~m}^{-3}$ in the PAL, the ionic
Debye length is on the order of a few $\mathrm{nm}$. Such a short Debye length means that the narrow Debye layers predicted by the surface-polarization model may be difficult to resolve experimentally due to lower limits on the spatial resolution of KPFM of 20-30 nm [39]. However, there is good agreement between the model and published KPFM data that, rather than a single diode junction, there are significant potential drops across both PAL-TL interfaces in a PSC.

Previously, theoretical studies of surface-polarization models have focused on explaining the origin of the problematic $J-V$ hysteresis exhibited by PSCs $[33,37,47]$. Other unusual characteristics, which have been encountered when using alternative characterization techniques to assess PSCs, such as electrochemical impedance spectroscopy (EIS) have also been studied [48]. In this work, we focus exclusively on the steady-state performance of PSCs. Steady-state performance provides the most accurate assessment of the true potential of PSCs. Here, the term steady state is used to describe measurements in which changes in applied voltage are sufficiently slow that the potential distribution is continuously in equilibrium with the experimental conditions, i.e., the electric field in the bulk of the PAL is completely screened by the potential drops $V_{1-4}$ throughout the measurement.

The potential drops that lie within the perovskite $\left(V_{2}\right.$ and $V_{3}$ in Fig. 2) correspond to an accumulation of predominantly ionic charge, while the potential drops in the electron transport layer (ETL) and hole transport layer (HTL) ( $V_{1}$ and $V_{4}$, respectively) correspond to an equal and opposite accumulation of electronic charge [33]. Therefore, the potential drops $V_{1-4}$ obey different capacitance relations for each Debye layer. The capacitance relations derived in Ref. [33] are restated in Appendix A. Note that even the two Debye layers that lie within the perovskite

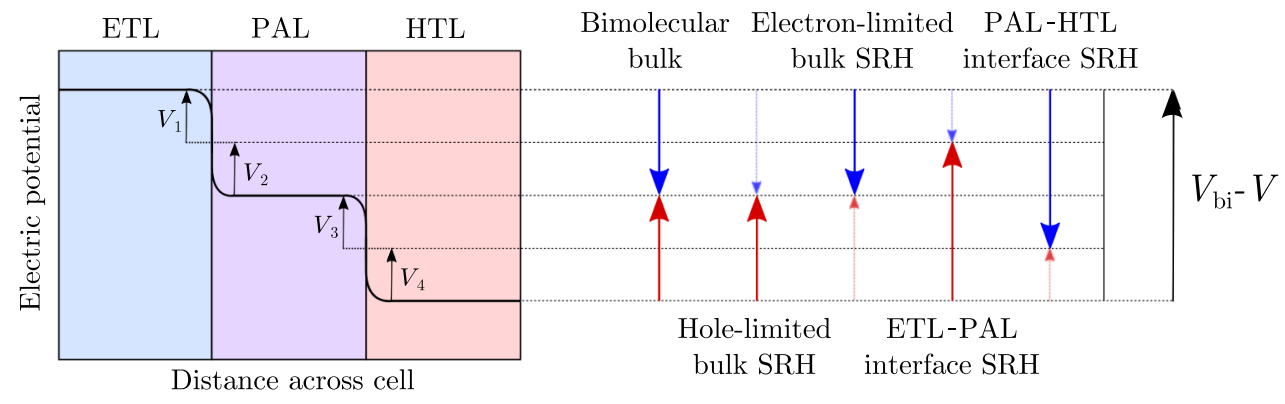

FIG. 2. Sketch showing the steady-state potential distribution across a planar PSC, when held at short circuit, alongside a diagram of the five different recombination processes considered in this work. When an electric field exists across the bulk of the perovskite absorber layer, the mobile ionic charge that exists within the layer migrates until sufficient charge has accumulated adjacent to the PAL-TL interfaces to compensate the potential difference across the cell. Therefore, at steady state, the electric field within the bulk of the PAL is zero (i.e., the potential is flat) and the potential difference across the cell is taken up solely within the four potential drops (labeled $V_{1-4}$ ) either side of each of the PAL-TL interfaces. For each of the recombination processes, the downwards-pointing blue arrow represents the potential energy to be overcome by an electron from the external circuit, while the upwards-pointing red arrow represents the same for a hole, in order for pair of charge carriers to recombine. The larger arrows indicate the processes, which limit, and therefore control, the rates of recombination. 
exhibit different capacitance relations because ionic charge accumulates on one side of the perovskite but becomes depleted on the other. While a high density of ionic charge can accumulate in a narrow region, there is a limit on the amount of charge that can be depleted per unit volume and so the depleted Debye layer can be much wider (resulting in a larger potential drop) than the corresponding accumulation layer. The values of $V_{1-4}$ can be determined from the four capacitance relations, a conservation law for the amount of ionic charge and the steady-state assumption that the sum of $V_{1-4}$ equals the total potential drop across the cell, $V_{\mathrm{bi}}-V$ (where $V_{\mathrm{bi}}$ is the cell's built-in voltage, defined as the difference in workfunction between the two TLs), as detailed in Appendix A. The relative sizes of $V_{1-4}$ vary depending on the applied voltage. In Fig. 3, we show how the total potential drop across the cell is divided between the four potential drops $V_{1-4}$ during a steady-state $J-V$ scan of Example Cell A, whose parameter values are listed within the Supplemental Material [49].

Furthermore, using KPFM, Cai et al. [40] show that the proportion of the total potential drop that falls across each PAL-TL interface (i.e., the relative sizes of $V_{1-4}$ in Figs. 2 and 3) can vary due to changes in the physical properties of the device. This result is in agreement with findings from device modeling by Courtier et al. [33], which explain how the values of the permittivity and the mean density of the majority mobile charge species in each layer have a dominant effect on the relative sizes of the potential drops. In particular, the larger the product of these two values, the smaller the potential drop located within the relevant material, and vice versa. This effect is a consequence of Gauss's law for the electric potential. In Fig. 3, the largest fraction of the total potential drop lies within the HTL, in $V_{4}$, because the HTL has a lower permittivity and a lower majority carrier density than the other two layers. This is expected to be the case for PSCs made with an organic hole-transporting material such as spiro-OMeTAD.

Based on the form for the potential distribution shown in Fig. 2, an analytic model for the steady-state $J-V$ characteristics of a PSC can be derived in an analogous way to the classical diode equation. By applying an appropriate choice of simplifications to the surface-polarization model from Ref. [33], an analytic expression for the steady-state current density $J$ in terms of the potential differences $V_{1-4}$ can be derived. Previously, for a single-layer model of a PSC, Richardson and coworkers [37] derived an analytic expression for the current density for a cell limited by holedominated SRH recombination within the PAL. Here, we consider the three core layers of a PSC and five different forms of recombination.

The simplifying assumptions applied to the surfacepolarization model that is presented in Sec. 3.5 of [33] are as follows. The assumptions are chosen in line with parameter estimates used in our previous work [33].
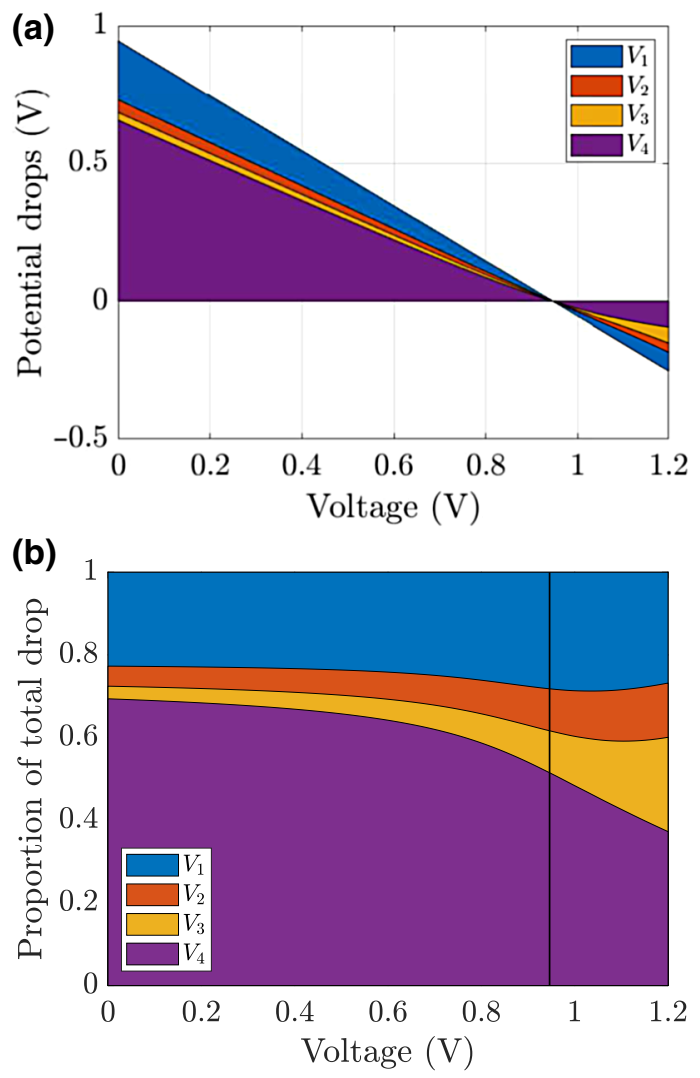

FIG. 3. Comparison of the sizes of the four potential drops $V_{1-4}$ (see Fig. 2) versus applied voltage during a steady state $J-V$ scan of Example Cell A, calculated from numerical solution of the capacitance relations previously used in Ref. [33] and restated in Eqs. (A4)-(A6) in Appendix A. (a) Stacked line chart of $V_{1-4}$ versus applied voltage. (b) Stacked line chart of the proportion of the total potential drop contained within each Debye layer, calculated as $V_{i} /\left(V_{\mathrm{bi}}-V\right)$ for $i=1$ to 4 . The vertical black line indicates the position of $V=V_{\mathrm{bi}}$.

Firstly, the steady-state simplification is encapsulated in the assumption that the electric field within the bulk of the PAL $\left(E_{\text {bulk }}\right)$ is always equal to zero. Secondly, it is assumed that the PSC is an efficient device in which the process of diffusion across the PAL dominates over the loss of charge carriers via recombination, when the cell is operating at applied voltages between $0<V<V_{\mathrm{OC}}$. The basis for this assumption is the exceptionally long carrier diffusion lengths exhibited by the types of perovskite, which are commonly employed in PSCs [50-52]. It is further assumed that the cell is held only under a moderate illumination intensity (up to approximately 1-Sun equivalent intensity). Lastly, it is assumed that the rate of recombination within a PSC is dominated by just one recombination mechanism, located either at one of the PAL-TL interfaces or in the bulk of the PAL, and that this rate can be well approximated by either a monomolecular or (in the case of band-to-band recombination) a bimolecular recombination rate. A systematic application of these assumptions to 
the equations that form the surface polarization model in Ref. [33] is deferred to Appendix A. The simplifications are verified against numerical simulations of a fully coupled charge-transport model. For details of the numerical method, see Sec. III B.

The result of systematically applying these assumptions is the following equation for the current density, which we term the ectypal diode equation for steady-state operation:

$$
J(V)=J_{s}-J_{d} \exp \left(\frac{V-V_{\mathrm{bi}}}{n_{\mathrm{ec}} V_{T}}\right), \quad n_{\mathrm{ec}}=\frac{V_{\mathrm{bi}}-V}{F(V)},
$$

where $J_{s}$ is the current density due to photogeneration, $J_{d}$ is a constant of proportionality for the current density lost to recombination and, in analogy with the ideality factor for the nonideal diode equation, we introduce $n_{\mathrm{ec}}$ as the ectypal factor. This voltage-dependent, dimensionless factor depends on the dominant pathway for recombination taking place within a device, via its dependence on the function $F(V)$. The function $F(V)$ captures the effect of ionic accumulation on the rate of recombination.

The value of $J_{s}$ depends only on the illumination intensity $I_{s}$ and light-absorption properties of the PAL, while the value of $J_{d}$ depends only on material properties of the device, see Table V. The function $F(V)$ depends only on the voltage-dependent Debye layer potential drops $V_{1-4}$, see Table I. In physical terms, $F(V)$ equals the potential difference, which forms a potential barrier to the recombination of charge carriers, at a given applied voltage. The ectypal factor $n_{\mathrm{ec}}$ is the reciprocal of the proportion of the total potential drop that forms a barrier to the dominant pathway for recombination, and is therefore expected to take positive values greater than 1 . Five example cases are listed in Table I. For details of how to evaluate the potential drops $V_{1-4}$ and hence calculate the current density expressed in Eq. (5), see Appendix 4.

Notably, Eq. (5) takes the same form as the approximate Shockley diode equation for positive applied voltages $\left(V \gg V_{T}\right)$ stated in Eq. (2). However, Eq. (5) incorporates an important modification in the form of the ectypal factor, which relates the performance of a PSC to the performance-limiting recombination mechanism and the internal distribution of the electric potential resulting from the accumulation of ionic charge within the PAL.

In addition to the mathematical derivation presented in Appendix A, a simple intuitive explanation for the results in Table I is provided as follows. When a PSC is in operation, current is generated by electrons entering the cell via the anode (by extracting a hole), traveling through the HTL and into the perovskite where they can be excited into the conduction band via the absorption of light. Free electrons are then extracted through the ETL and the cathode to complete the circuit. Current is also lost due to charge-carrier recombination within the cell. Note that recombination currents flow in the opposite direction to the photogenerated current. The total current is, therefore, the difference between the photogenerated current and the recombination current. The amount of recombination depends on the number of available charge carriers and the likelihood of recombination via a particular pathway. In the case of radiative recombination within the $\mathrm{PAL}$, the rate of recombination takes a bimolecular form $(R \approx \beta n p)$ as it depends on the availability of both a free electron and a hole. Therefore, the potential energy barrier to recombination $F(V)$ is equal to the total potential drop across the cell and so $n_{\mathrm{ec}}=1$. Note that this result is equivalent to the ideal case in the classical diode theory. In contrast, nonradiative recombination mechanisms (e.g., recombination via trap states) are often modeled using monomolecular rates. This is because the rate of recombination is limited only by the availability of one type of charge carrier. Therefore, the potential energy barrier to monomolecular-type recombination is just the potential difference between the external circuit and the location at which the recombination takes place, see Fig. 2. For example, for electron-limited, trap-assisted recombination at the PAL-HTL interface $\left(R_{r} \approx v_{n, H} n\right)$, the potential energy barrier $F(V)$ that the electrons must overcome [53] is the sum of the potential drops between the cathode and the PALHTL interface, i.e., $F(V)=V_{1}+V_{2}+V_{3}$. More examples are given in Table I.

Informally, we here assume that the ectypal factor can be estimated from experimental data using the standard methods of characterization, namely the dark $J-V$ and Suns- $V_{\mathrm{OC}}$ methods. However, unlike the ideality factor, the ectypal factor is by definition a function of the applied voltage and so it is necessary to justify this approach. In fact, analyzing the dark current density in Eq. (5) using the standard dark $J-V$ method does not return the ectypal factor but leads to

$$
\frac{1}{V_{T}}\left(\frac{\partial \ln J_{\mathrm{dark}}}{\partial V}\right)^{-1}=n_{\mathrm{ec}} \times\left[1-\frac{\left(V-V_{\mathrm{bi}}\right)}{n_{\mathrm{ec}}} \frac{d n_{\mathrm{ec}}}{d V}\right]^{-1} .
$$

We refer to the value of this expression as the measured ectypal factor. From this expression, it is clear that the measured ectypal factor is approximately equal to the true ectypal factor when the applied voltage is close to the built-in voltage (i.e., when $V-V_{\text {bi }} \approx 0$ ).

Similarly, the Suns- $V_{\mathrm{OC}}$ method also returns the measured ectypal factor:

$$
\frac{1}{V_{T}} \frac{\partial V_{\mathrm{OC}}}{\partial \ln F_{\mathrm{ph}}}=n_{\mathrm{ec}} \times\left[1-\frac{\left(V_{\mathrm{OC}}-V_{\mathrm{bi}}\right)}{n_{\mathrm{ec}}} \frac{d n_{\mathrm{ec}}}{d V_{\mathrm{OC}}}\right]^{-1} .
$$

As before, this method relies on the classical assumption that the short-circuit current density is directly proportional to the illumination intensity and the dominant recombination mechanism does not vary with illumination. 
TABLE I. Table showing the analytic expressions for the potential barrier $F(V)$ and the ectypal factor $n_{\mathrm{ec}}$ for five different recombination rates, see Fig. 2. Note that, at steady state, each of the potential drops $V_{1-4}$ is a function of the applied voltage $V$ (while the built-in voltage $V_{\mathrm{bi}}$ is a constant) and the sum of the four potential drops $V_{1-4}$ equals the total potential difference across the cell, $V_{\mathrm{bi}}-V$. The ectypal factor is defined in terms of the potential barrier $F(V)$ in Eq. (5b). The recombination parameters $\beta, \tau_{p}, \tau_{n}, v_{p, E}$, and $v_{n, H}$ are all constants of proportionality derived from the full forms of the recombination rates given in Table II.

\begin{tabular}{lcccc}
\hline \hline Type of recombination & Abbrev. & Approximate & Potential barrier & Ectypal factor \\
& & recombination rate & $n_{\mathrm{ec}}$ & 1 \\
\hline Bimolecular bulk recombination & $\mathrm{Rb}$ & $R \approx \beta n p\left(\mathrm{~m}^{-3} \mathrm{~s}^{-1}\right)$ & $V_{1}+V_{2}+V_{3}+V_{4}$ & $\frac{V_{\mathrm{bi}}-V}{V_{\mathrm{bi}}-V_{-}-V_{1}-V_{2}}$ \\
Hole-limited bulk SRH & $\mathrm{Rp}$ & $R \approx \frac{p}{\tau_{p}}\left(\mathrm{~m}^{-3} \mathrm{~s}^{-1}\right)$ & $V_{3}+V_{4}$ & $\frac{V_{\mathrm{bi}}-V}{V_{\mathrm{bi}}-V_{-}-V_{3}-V_{4}}$ \\
Electron-limited bulk SRH & $\mathrm{Rn}$ & $R \approx \frac{n}{\tau_{n}}\left(\mathrm{~m}^{-3} \mathrm{~s}^{-1}\right)$ & $V_{1}+V_{2}$ & $\frac{V_{\mathrm{bi}}-V}{V_{\mathrm{bi}}-V-V_{1}}$ \\
ETL-PAL interface SRH & $\mathrm{Rl}$ & $R_{l} \approx v_{p, E} p\left(\mathrm{~m}^{-2} \mathrm{~s}^{-1}\right)$ & $V_{2}+V_{3}+V_{4}$ & $\frac{V_{\mathrm{bi}}-V}{V_{\mathrm{bi}}-V-V_{4}}$ \\
PAL-HTL interface SRH & $\mathrm{Rr}$ & $R_{r} \approx v_{n, H} n\left(\mathrm{~m}^{-2} \mathrm{~s}^{-1}\right)$ & $V_{1}+V_{2}+V_{3}$ & \\
\hline \hline
\end{tabular}

Hence, we find that both characterization methods return values for the measured, rather than true, ectypal factor, but that these values coincide for applied voltages close to the built-in voltage. In Sec. IV, we compare values for both the measured and true ectypal factors (in Fig. 6) in order to justify our use of the standard methods to analyze the current-voltage characteristics of PSCs.

Note that external series and shunt resistance can be incorporated into the ectypal diode equation in an analogous way to how they are incorporated into the standard diode equation, see Appendix B. However, for highefficiency cells, parasitic resistances should have a minimal influence on the $J-V$ characteristics of a cell operating in its power-generating regime. Therefore, we do not investigate the influence of parasitic resistances in this work.

\section{B. Numerical simulation method}

In order to verify the results of our analytical model, we perform numerical simulations of a fully coupled, timedependent charge-transport model of a PSC that includes the migration of one type of mobile ionic charge within the PAL. Similar models have successfully been shown to reproduce many features of the $J-V$ hysteresis exhibited by PSCs $[33,34]$. To perform the simulations, we use the open-source IonMonger simulation tool described in detail in Ref. [43]. The model consists of a full set of drift-diffusion equations for the ion vacancy, electron and hole densities, coupled to Poisson's equation for the electric potential. The numerical method upon which this code is based is a combination of a finite-element discretization in space and the adaptive time-stepping provided by MATLAB's differential-algebraic equation solver ode15s [54]. The accuracy and speed of this method, in comparison to two alternative methods of solution, are demonstrated in
Ref. [55]. A list of the parameter values used in this work is provided within the Supplemental Material [49].

The measurement protocol for all $J-V$ curves in this work consists of a 60 -s preconditioning step at $1.2 \mathrm{~V}$, followed by scanning the voltage down to short circuit $(0 \mathrm{~V})$ and back. The numerical simulations use a scan rate of $1 \mathrm{mV} \mathrm{s}^{-1}$ (except in Fig. 4 where the rate is $100 \mathrm{mV} \mathrm{s}^{-1}$ ). A scan rate of $1 \mathrm{mV} \mathrm{s}^{-1}$ is assumed to be slow enough to approximate the steady-state behavior modeled by the ectypal diode equation, in which the ionic charge is in continuous equilibrium with the applied voltage. All simulations are performed either in the dark or in the light at an illumination intensity equal to the equivalent of 0.25 , 0.5 , or 1 Sun using an incident photon flux of $1.4 \times$ $10^{21} \mathrm{~m}^{-2} \mathrm{~s}^{-1}$.

In Fig. 4, we use the IonMonger simulation tool to demonstrate that a PSC model that includes one species of mobile ionic charge within the PAL is able to predict voltage-dependent values for the "ideality factor," similar to those observed by experiment $[3,6,24,25]$. These results show that the origin of this anomalous behavior is not a voltage-dependent series or shunt resistance, as suggested in Ref. [6], but a result of the intrinsic, mixed electronicionic response of a PSC. Crucially, unlike the standard theory, our alternative, analytic model captures the key effects of ionic accumulation within a PSC via the form of the voltage-dependent ectypal factor.

Figure 5(a) shows very good agreement, over many orders of magnitude, between $J-V$ curves calculated using the ectypal diode equation and numerical solutions of the full model, both in the dark and in the light. Note that the ectypal diode equation depends on obtaining a numerical solution for the value of the four Debye layer potential drops $V_{1-4}$, which depend on the capacitance relations from Ref. [33] and restated in Appendix A. For details of how to evaluate the ectypal diode equation, see 
(a)

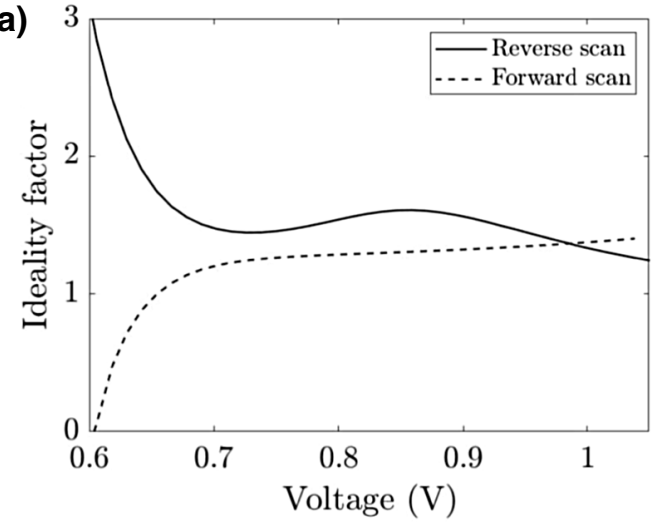

(b)

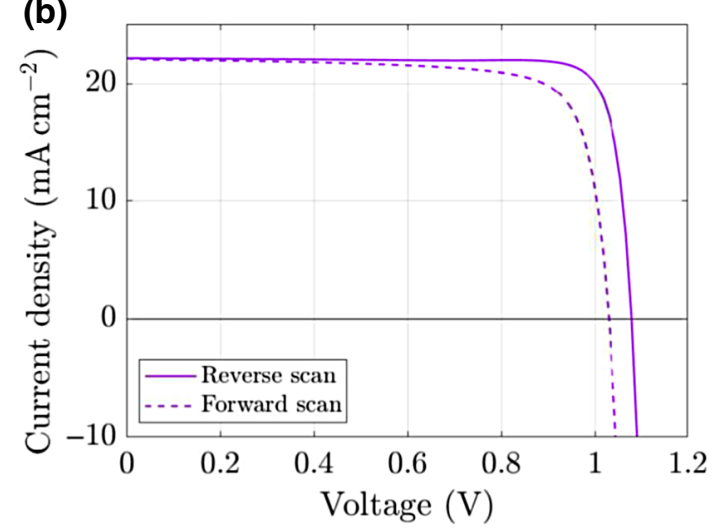

FIG. 4. Example of the unusual $J-V$ characteristics of a PSC, reproduced by numerical simulations of a time-dependent charge-transport model for ion migration and charge-carrier transport using the IonMonger simulation tool [43]. (a) The "ideality factor" computed using the dark $J-V$ method, described in Sec. II, from a simulation of the reverse scan and subsequent forward scan of a $100 \mathrm{mV} \mathrm{s}^{-1} J-V$ measurement of Example Cell A limited by recombination at the ETL-PAL interface performed in the dark. (b) The reverse and forward scans of a $100 \mathrm{mV} \mathrm{s}^{-1} J-V$ scan simulated using the same set of parameter values except that it is performed in the light at an illumination intensity equivalent to 1 Sun.

Appendix 4. The good agreement between the ectypal diode equation and the numerical simulations relies on the accurate description of the steady-state distribution of the potential provided by these capacitance relations. Figure 5(b) also shows very good agreement between numerical solutions and the ectypal diode equation for steady-state $J-V$ curves measured under three different levels of illumination up to the equivalent of 1 Sun. In particular, this figure shows that the ectypal diode equation is able to accurately predict the relationship between the open-circuit voltage and the illumination intensity. This accuracy justifies our use of the ectypal diode equation to analyze Suns- $V_{\mathrm{OC}}$ measurements.

\section{RESULTS}

In this section, we validate the ectypal diode equation against full numerical solutions to a charge transport model
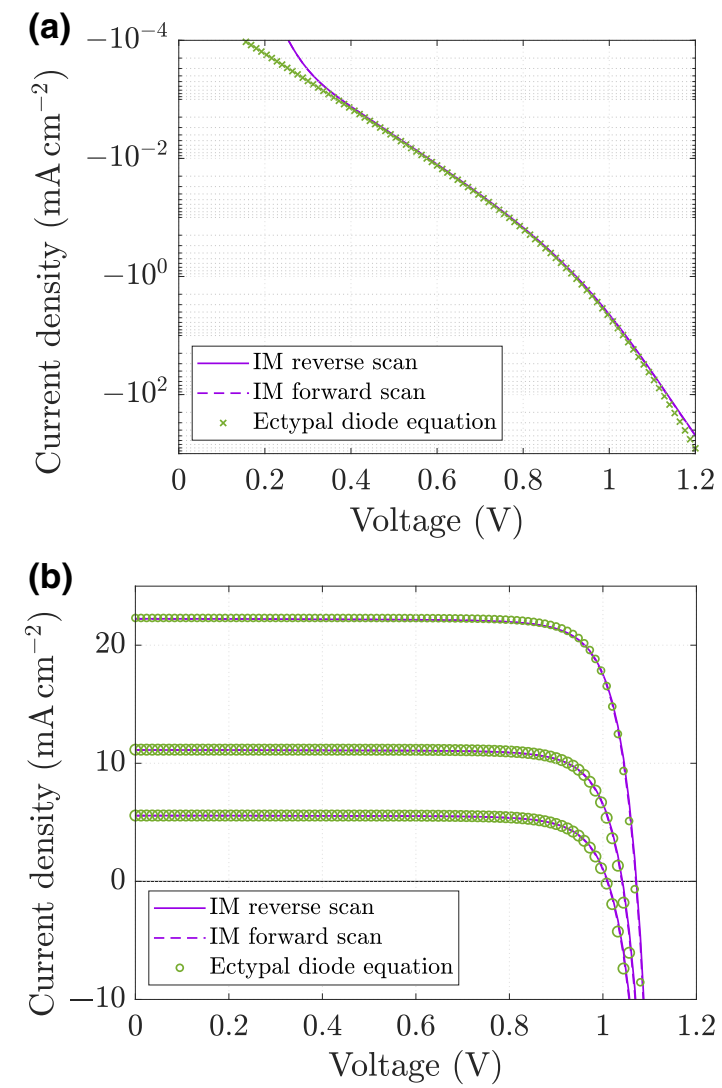

FIG. 5. Validation of the ectypal diode equation against full numerical drift-diffusion simulations of Example Cell A limited by recombination at the PAL-HTL interface with the associated parameter values given in Table II. The numerical solutions are obtained using the IonMonger simulation tool [43] and indicated by the abbreviation "IM." (a) The steady-state current density (plotted on a logarithmic scale) versus applied voltage in the dark. (b) The steady-state current density vs applied voltage at light intensities equivalent to $0.25,0.5$, and 1 Sun illumination.

for ion vacancy motion and charge-carrier transport across a PSC [33]. We illustrate how the ectypal diode equation can be applied to data from numerical simulations, which we regard as a proxy for data obtained from real cells. Values of the ectypal factor, corresponding to each of five different recombination mechanisms, are obtained in one of the following ways:

(a) The true ectypal factor is the reciprocal of the proportion of the total potential drop that forms a barrier to recombination (see Fig. 2), calculated from the relevant expression for $F(V)$ from Table I and the solution of Eqs. (A4)-(A6) for the potential drops $V_{1-4}$.

(b) The theoretical, measured ectypal factor is calculated from the current density predicted by the ectypal diode equation in Eq. (5) using either the dark $J-V$ method via Eq. (6) or the Suns- $V_{\mathrm{OC}}$ method via Eq. (7). These values also depend on the relevant form of $F(V)$ and the 
TABLE II. Table showing the five different recombination mechanisms, associated parameter values and representative results obtained using the two different methods of analysis considered in this work. Only one mechanism is included in each simulation. The full forms of the recombination rates $^{\mathrm{a}}$ are used in the numerical simulations, while the ectypal diode equation depends on the approximate forms given in Table I. The representative values of the measured $n_{\mathrm{ec}}$ are those for Example Cell A, which is described by the parameter values listed within the Supplemental Material [49]. The representative value from the dark $J-V$ analysis is the average of the measured $n_{\mathrm{ec}}$ corresponding to applied voltages between $0.7-1.0 \mathrm{~V}$, see Fig. 6 . The representative value for the Suns- $V_{\mathrm{OC}}$ technique is determined from a linear fit to the data for illumination intensities between $10^{-3}$ and $10^{-1}$ Suns, see Fig. 7 .

\begin{tabular}{|c|c|c|c|c|}
\hline Type of recombination & Abbrev. & $\begin{array}{l}\text { Full form of the } \\
\text { recombination rate }\end{array}$ & $\begin{array}{l}\text { Parameter } \\
\text { values }\end{array}$ & $\begin{array}{l}\text { Representative } n_{\mathrm{ec}} \text { from } \\
\text { dark } J-V-\text { Suns- } V_{\mathrm{OC}}\end{array}$ \\
\hline Bimolecular bulk recombination & $\mathrm{Rb}$ & $R=\beta\left(n p-n_{i}^{2}\right)$ & $\beta=10^{-11} \mathrm{~m}^{3} \mathrm{~s}^{-1}$ & $1.0-1.0$ \\
\hline Hole-limited bulk SRH & $\mathrm{Rp}$ & $R=\frac{n p-n_{i}^{2}}{\tau_{n} p+\tau_{p} n+c_{1}}$ & $\tau_{n}=3 \times 10^{-11} \mathrm{~s} \tau_{p}=3 \times 10^{-9} \mathrm{~s}$ & $1.5-1.5$ \\
\hline Electron-limited bulk SRH & $\mathrm{Rn}$ & $R=\frac{n p-n_{i}^{2}}{\tau_{n} p+\tau_{p} n+c_{2}}$ & $\tau_{n}=3 \times 10^{-8} \mathrm{~s} \tau_{p}=3 \times 10^{-10} \mathrm{~s}$ & $2.7-2.8$ \\
\hline ETL/PAL interface SRH & $\mathrm{Rl}$ & $R_{l}=\frac{n p-n_{i}^{2}}{\frac{p}{v_{n, E}}+\frac{n}{v_{p, E}}+c_{3}}$ & $v_{n, E}=10^{5} \mathrm{~ms}^{-1} v_{p, E}=30 \mathrm{~m} \mathrm{~s}^{-1}$ & $1.4-1.4$ \\
\hline PAL/HTL interface SRH & $\mathrm{Rr}$ & $R_{r}=\frac{n p-n_{i}^{2}}{\frac{p}{v_{n, H}}+\frac{n}{v_{p, H}}+c_{4}}$ & $v_{n, H}=30 \mathrm{~m} \mathrm{~s}^{-1} v_{p, H}=10^{5} \mathrm{~m} \mathrm{~s}^{-1}$ & $2.6-2.7$ \\
\hline
\end{tabular}

${ }^{\text {a }}$ The definitions of the symbols are as follows: $\beta$ is the constant of proportionality for bimolecular bulk recombination; $n_{i}$ is the intrinsic carrier concentration in the perovskite; $\tau_{n}$ and $\tau_{p}$ are the electron and hole pseudolifetimes for SRH in the bulk, respectively; $v_{n}$ and $v_{p}$ are the SRH recombination velocities for electrons on the left and holes on the right, respectively, of the interface between the PAL and the TL denoted by the second subscript $(E$ or $H)$; and $c_{1-4}$ are constants whose magnitudes are negligibly small. The carrier concentrations ( $n$ and $p$ ) in the two interface recombination rates are the concentrations on opposite sides of the interface (electrons on the left, holes on the right).

solution of Eq. (A4)-(A6) for the potential drops $V_{1-4}$ (see Appendix 4).

(c) The simulated, measured ectypal factor is calculated from the current density obtained from numerical simulations of the full charge-transport model, using either the dark $J-V$ method via Eq. (6) or the Suns- $V_{\text {OC }}$ method via Eq. (7). The simulations are performed using the IonMonger simulation tool [43] (see Sec. III B).

In line with the classical theory of solar cells, we assume that just one recombination mechanism dominates the observed behavior of a PSC. The five different recombination mechanisms we consider are listed in Table II. This table also provides a representative value of the simulated, measured ectypal factor obtained using the dark $J-V$ method and Suns- $V_{\mathrm{OC}}$ method for Example Cell $\mathrm{A}$ in each of the five cases. These representative values correspond to the values that would usually be referred to as ideality factors.

\section{A. Dark $J-V$ method}

In Fig. 6, we show the results of our dark $J-V$ analysis of Example Cell A (which has a mean ion vacancy density of $N_{0}=1.6 \times 10^{25} \mathrm{~m}^{-3}$ in the PAL). For each of five recombination mechanisms, we compare the measured ectypal factor as determined from full numerical simulations and the measured and true ectypal factors predicted by the ectypal diode theory. The results for the three types of bulk recombination are shown in (a), while those for the two types of interface recombination are shown in (b). The dark $J-V$ curves corresponding to each of the recombination mechanisms, for both Example Cells A and B, are shown in Fig. S1 within the Supplemental Material [49].

From the results in Fig. 6, it becomes apparent that the classical diode theory cannot be used to correctly diagnose the limiting recombination mechanism from the dark $J-V$ data. Even in the ideal case where a single recombination mechanism controls the observed behavior, the measured "ideality" factor is not constant with respect to the applied voltage. The ectypal factor is intrinsically voltage dependent and the values are different from those predicted by the classical theory, with one exception. The only recombination mechanism whose characteristics remain the same, between the classical diode theory and the proposed ectypal theory, is that of bimolecular bulk recombination, which displays a constant value of 1 . For the other four recombination mechanisms, the nonlinearity of the ectypal factor leads to a difference between the values of the measured and true values of the ectypal factor, due to the derivative term in Eq. (6). The effect of this extra derivative term is to exaggerate the nonlinearity of the ectypal factor. However, the measured ectypal factors still show similar 

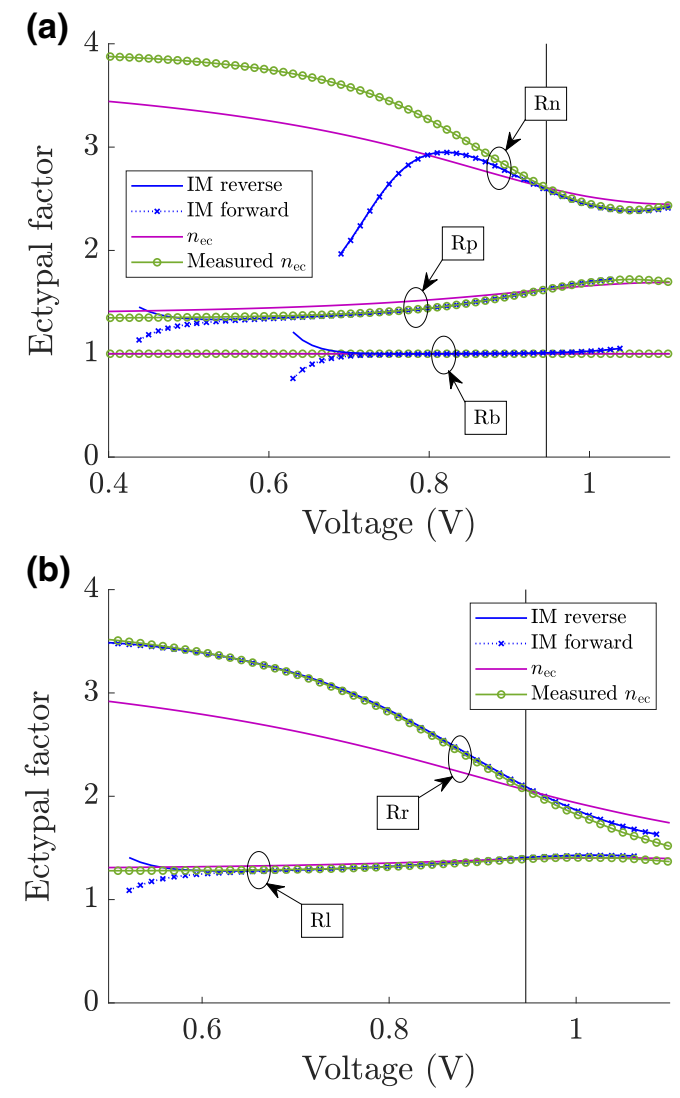

FIG. 6. Simulated and theoretical results for the ectypal factor versus applied voltage, determined using the dark $J-V$ method, for Example Cell A limited by each of the five recombination mechanisms listed in Tables I and II. Panel (a) shows the ectypal factor versus voltage corresponding to each of the three different types of bulk recombination, while (b) shows the results of the two types of interface recombination. Simulated values of the measured ectypal factor (indicated by the abbreviation "IM") are calculated (simulated values are shown only where the current densities at neighboring points on the $J-V$ curve (with $12-\mathrm{mV}$ spacing) are between $10^{-6}$ and $10 \mathrm{~mA} \mathrm{~cm}^{-2}$ apart, or between $4 \times 10^{-3}$ and $10 \mathrm{~mA} \mathrm{~cm}^{-2}$ apart in the case of "Rn." The deviation of the simulated values from the theoretical measured $n_{\mathrm{ec}}$ at low voltages is due to the sensitivity of the calculation on the very small and nonsteady carrier concentrations simulated at a scan rate of $1 \mathrm{mV} \mathrm{s}^{-1}$; except in the case of "Rn," where the deviation is due to a breakdown in the assumption that the SRH recombination rate can be approximated by a monomolecular rate) using Eq. (6). Theoretical values of the true and measured ectypal factors are calculated from Eqs. (6) and (5b), respectively, using the values of $V_{1-4}$ plotted in Fig. 3.

trends and values to the true $n_{\mathrm{ec}}$ and so the following deductions can be made from Fig. 6 .

For this particular cell (Example Cell A), at applied voltages below $1 \mathrm{~V}$, the majority of the potential difference is taken up by the potential drop across the Debye layer within the HTL $\left(V_{4}\right)$, as shown in Fig. 3. This means that more than half of the total potential difference across the cell acts as a barrier to recombination mechanisms that rely on holes entering the cell from the external circuit, e.g., $R_{p}$ and $R_{l}$. Therefore, (recalling that the ectypal factor is the reciprocal of the proportion of the total potential drop that forms a barrier to recombination) the ectypal factors corresponding to these hole-limited recombination rates take values less than 2 . On the other hand, less than half of the total potential difference forms a barrier to electrons entering the cell from the external circuit. Therefore, the electron-limited recombination rates, $R_{n}$ and $R_{r}$, return values that are greater than 2 . As the voltage is increased above $1 \mathrm{~V}$, the proportion of the potential drop that lies within $V_{4}$ decreases and so more than half of the total potential difference lies in the sum of $V_{1}+V_{2}+V_{3}$. As a result, the value of the ectypal factor for $R_{r}$ decreases below 2 as the voltage increases above $1 \mathrm{~V}$. Note that these results are not generic and the interpretation of ectypal factors must be guided by accurate knowledge of the distribution of the electric potential across the PSC under investigation.

For Example Cell A, it is notable that the potential drops that lie within the PAL $\left(V_{2}\right.$ and $\left.V_{3}\right)$ are small relative to those that lie within the TLs $\left(V_{1}\right.$ and $\left.V_{4}\right)$. As a result, it is possible to distinguish between cells that are dominated by bimolecular bulk recombination $\left(n_{\mathrm{ec}}=\right.$ $1)$, hole-limited recombination mechanisms $\left(1<n_{\mathrm{ec}}<2\right)$ or electron-limited recombination mechanisms $\left(n_{\mathrm{ec}}>2\right)$. However, for cells limited by the injection of one type of carrier, it is difficult to determine the location of the recombination, i.e., it is difficult to distinguish between $R_{p}$ and $R_{l}$, or alternatively between $R_{n}$ and $R_{r}$. Furthermore, this is not the case for all cells, as demonstrated later with the help of Fig. 8. The deductions that can be made from the value of the ectypal factor, depend on the distribution of the potential between the four Debye layers.

Next we investigate whether the second standard technique for analyzing the performance of solar cells, namely the Suns- $V_{\mathrm{OC}}$ method, is able to return the same diagnosis as the dark $J-V$ method, for the same parameter values, when using the ectypal diode theory.

\section{B. Suns- $V_{\text {OC }}$ method}

In Fig. 7, we show the results of a Suns- $V_{\mathrm{OC}}$ analysis of Example Cell A in the five cases where it is limited by one of the five recombination mechanisms listed in Table II. This plot demonstrates that the different recombination mechanisms correspond to different values of the representative value of the measured ectypal factor (that which would usually be referred to as an ideality factor). The representative value of the ectypal factor is calculated from a linear fit to the measured ectypal factor corresponding to the 17 simulations performed at light intensities between (and including) $10^{-3}$ and $10^{-1}$ Suns. These values are listed alongside representative values estimated from our 

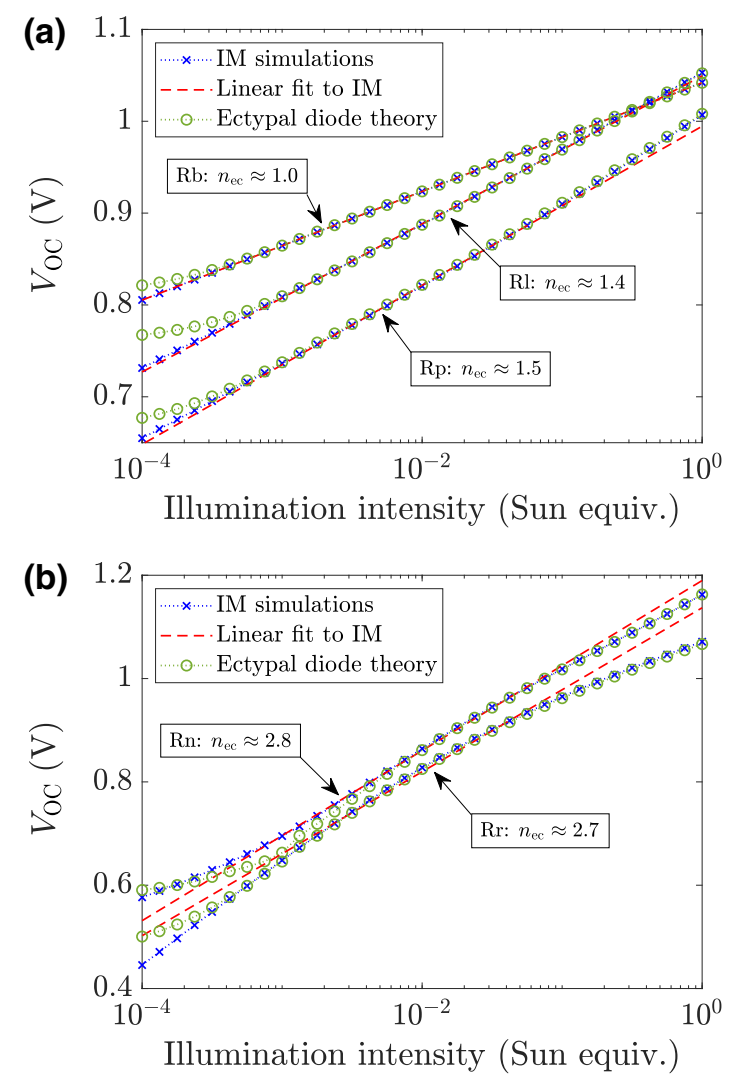

FIG. 7. Simulated, fitted, and theoretical results for a Suns$V_{\mathrm{OC}}$ measurement of Example Cell A for each of the five recombination mechanisms listed in both Tables I and II. The abbreviation "IM" indicates the results obtained using the IonMonger simulation tool [43]. The representative values of the measured ectypal factor (shown in the labels) are obtained from linear fits to the points corresponding to values of the illumination intensity between $10^{-3}$ and $10^{-1}$ Suns via Eq. (7). Panel (a) shows the results of recombination mechanisms, which display a representative value between 1 and 2, while (b) shows the results of recombination mechanisms, which exhibit values greater than 2 .

dark $J-V$ analysis in the last column of Table II. Reassuringly, the two methods result in very similar values in each case. However, it can be difficult to distinguish between the different cases. It is also clear that, even in the ideal case where one mechanism controls the behavior of a cell, the ectypal factor is not constant with respect to the voltage across the cell. Nonlinear behavior can be seen in the lines corresponding to higher values of the ectypal factor in Fig. 7(b), although the representative value obtained from the linear fit remains a useful measure.

In Fig. 8, we move on to consider a second example: Example Cell B. The only difference between the cell parameters for Example Cells A and B is the value of the mean density of ion vacancies in the PAL. For A, $N_{0}=1.6 \times 10^{25} \mathrm{~m}^{-3}$, while for B, $N_{0}=5 \times 10^{23} \mathrm{~m}^{-3}$.
This difference results in a significant change to the distribution of the potential between the four Debye layers, as shown in (a) and (b) of Fig. 8. For Example Cell B, around half of the potential difference is taken up within the PAL, in the sum of $V_{2}+V_{3}$, while significantly less than half is taken up within each of the Debye layers in the TLs ( $V_{1}$ and $\left.V_{4}\right)$. Therefore, when the cell is limited by interface recombination, it exhibits values of the ectypal factor between $1<n_{\mathrm{ec}}<2$, while in the case of bulk SRH recombination $n_{\mathrm{ec}}>2$. As before, $n_{\mathrm{ec}}=1$ signifies bimolecular bulk recombination. These examples demonstrate how, in the case of planar PSCs, the interpretation of the results of standard techniques must be made with careful consideration of the potential distribution across a cell versus applied voltage. The ectypal diode theory set out in this work provides a framework for deducing the performance-limiting recombination mechanism in a particular PSC architecture.

\section{DISCUSSION}

Here, we discuss the implications of the ectypal diode theory proposed in this work on experimental results published in the existing literature on planar PSCs.

Tress et al. [6] calculate voltage-dependent "ideality factors" from dark $J-V$ measurements (measured at a scan rate of $20 \mathrm{mV} \mathrm{s}^{-1}$ ), which show significant hysteresis between the forward and reverse scans. These results cannot be interpreted using the ectypal diode theory because they do not correspond to the steady-state $J-V$ characteristics of the device. A Suns- $V_{\mathrm{OC}}$ analysis is also performed (using light intensities between $10^{-3}-10^{-1}$ Suns) from which they obtain an "ideality factor" of 1.6 for a planar PSC with a standard $\mathrm{SnO}_{2} /$ perovskite/Spiro-MeOTAD architecture. From this analysis and the high open-circuit voltage of $1.2 \mathrm{~V}$ for this cell, Tress et al. [6] conclude that the performance-limiting recombination mechanism is SRH recombination via defects in the bulk of the perovskite.

Since the distribution of the electric potential across a PSC has a significant impact on its steady-state $J-V$ behavior, it is difficult to draw conclusions from such data without knowing the distribution of the potential across the cell. However, the ectypal diode theory tells us that the dominant recombination mechanism of a cell with $n_{\mathrm{ec}}=1.6$ is one that is controlled by an energy barrier that is more than half of the total potential drop across the cell (because $1 / n_{\mathrm{ec}}>50 \%$ ). In order to deduce the location of the largest potential drop within the $\mathrm{SnO}_{2}$ /perovskite/SpiroMeOTAD device, we have to make some assumptions about the cell parameters. By assuming that the inorganic $\mathrm{SnO}_{2}$ ETL has a higher permittivity and effective doping density than the organic Spiro-MeOTAD HTL, we can assume that $V_{4}$ is larger than $V_{1}$ (as is the case for both Example Cells A and B) [33]. The fact that hysteresis is observed on a slow timescale indicates that a significant 

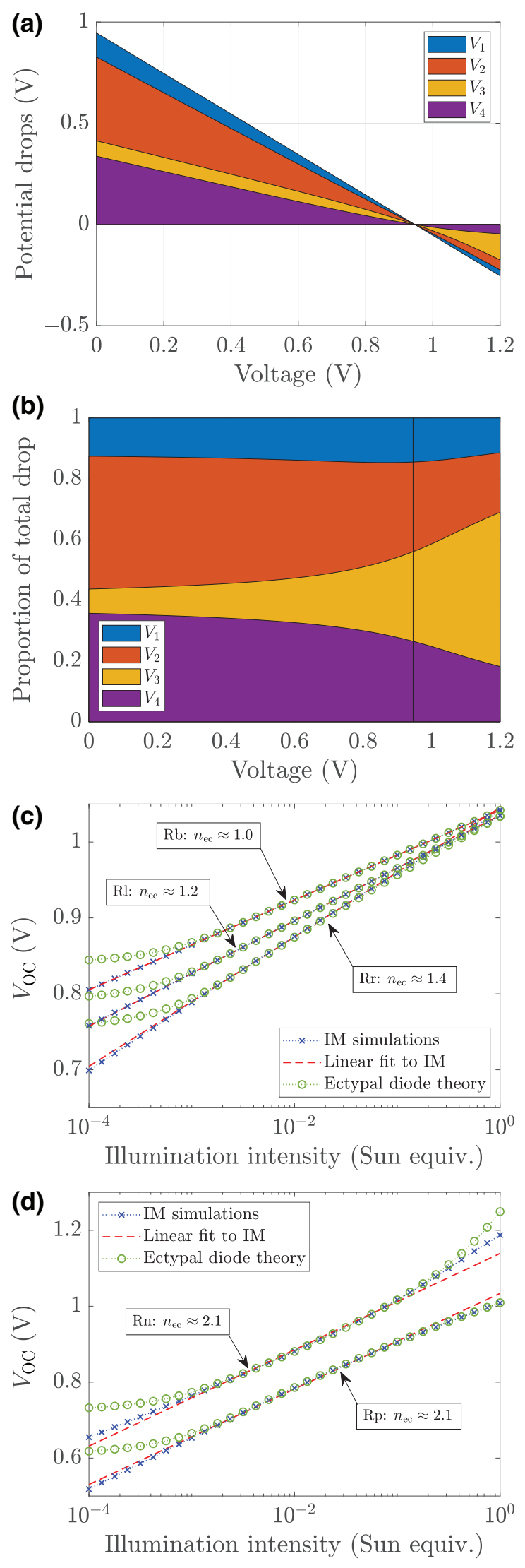

FIG. 8. (a),(b) Equivalent to Fig. 3 and (c),(d) equivalent to Fig. 7, but for Example Cell B. amount of ionic accumulation is likely to occur within the PAL and, therefore, that $V_{2}$ and $V_{3}$ are comparatively large. We therefore suggest that the potential distribution may resemble that of Example Cell B. If this is the case, an ectypal factor of $n_{\mathrm{ec}}=1.6$ could be attributed to electronlimited interface recombination occurring at the PAL-HTL interface. This is a different conclusion to that made by Tress et al. [6]. This highlights the importance of having an accurate knowledge of the potential distribution across a PSC in order to obtain an accurate diagnosis. Reliable diagnosis of the dominant recombination mechanism in a PSC is vital for informing future cell design.

In work by Caprioglio et al. [16], "ideality factors" between 1.2-1.5 are calculated from Suns- $V_{\mathrm{OC}}$ measurements (performed at light intensities between $10^{-2}-1$ Sun) for different types of PSC, which have the inverted architecture HTL/perovskite/C60. The cells employed either PTAA or P3HT as the HTL material. Caprioglio et al. [16] conclude that these values result from a single recombination mechanism in each cell, which is located at one of the PAL-TL interfaces. This conclusion is made despite the lack of an explanation from the classical diode theory for the noninteger values of the "ideality factor." If the potential distribution across these cells resembles that of Example Cell B, the results in Fig. 8 indicate that ectypal factors between $1<n_{\mathrm{ec}}<2$ can be attributed to dominant interface recombination (for which the energy barriers to recombination are more than half of the total potential drop across the cell). This diagnosis is consistent with the conclusions of Caprioglio et al. [16].

Cai et al. [40] present experimental results for a set of planar PSCs, which use the most common, standard architecture: compact- $\mathrm{TiO}_{2} / \mathrm{MAPI}$ perovskite/Spiro-OMeTAD. This study shows a clear trend between the distribution of the electric potential and the value of the "ideality factor." This trend is not explained by the classical diode theory but it can be explained using the ectypal diode theory as follows. The distribution of the electric field, as measured from KPFM, across a device is shown to depend on the ratio between the amount of $\mathrm{PbI}_{2}$ and MAI in the perovskite precursor solution. It is shown that a cell fabricated using a $\mathrm{PbI}_{2}$-rich precursor solution results in a large potential drop across the PAL-HTL interface and a small potential drop across the ETL-PAL interface (i.e., $\left.\left|V_{1}+V_{2}\right| \ll\left|V_{3}+V_{4}\right|\right)$, while the opposite is seen for a cell that uses an MAI-rich precursor solution. A dark $J$ $V$ analysis of a set of five cells with a smoothly varying $\mathrm{PbI}_{2}$ : MAI precursor ratio shows that the "ideality factor" decreases from approximately 2.5 to 1.4 as the potential shifts from the ETL-PAL interface $\left(V_{1}+V_{2}\right)$ to the PAL-HTL interface $\left(V_{3}+V_{4}\right)$. This shift corresponds to an increase in the magnitude of $V_{3}+V_{4}$ from less than half to more than half of the total potential drop, as a result of the increasing $\mathrm{PbI}_{2}$ : MAI precursor ratio. Assuming that a single recombination mechanism dominates the 
behavior of the cells, this trend implies that the dominant recombination mechanism is controlled by $V_{3}+V_{4}$, or possibly $V_{2}+V_{3}+V_{4}$. Therefore, we conclude that the performance of this PSC architecture is dominated by holelimited recombination located either in the bulk or at the ETL-PAL interface.

The dependence of the ectypal factor on the potential distribution across a PSC suggests that certain physical properties of a PSC can be tuned in order to reduce losses due to a particular type of recombination, or to change which recombination mechanism dominates. Increasing the permittivity or the effective doping density of one of the TLs can shift some of the potential drop across that layer to other layers of the cell [33], resulting in a similar trend to that observed by Cai et al. [40]. Note that the outcome of changing the properties of a TL may be counterintuitive because changing the properties of one TL may have a strong effect on the electric potential distribution across the whole cell. For example, increasing the doping density of the ETL reduces the potential drop $V_{1}$, but increases $V_{2-4}$, and therefore the rate of hole-limited interface recombination at the ETL-PAL interface may be reduced, while the rate of electron-limited interface recombination at the PAL-HTL is increased. For the dependence of the ectypal diode equation on other physical properties, see Table $\mathrm{V}$ for the definitions of the dark current density parameter $J_{d}$ for the five different recombination mechanisms considered in this work.

Finally, we note that the assumption of steady-state conditions is also expected to be applicable to small perturbation measurements, such as high-frequency EIS, in which changes in the external stimulus (applied voltage or illumination intensity) are sufficiently small and fast that the distribution of ions and corresponding potential drops $V_{1-4}$ can be assumed to remain in constant equilibrium with the average conditions during the measurement. This prediction is in agreement with work by Anta and coworkers $[15,23]$ who find that it is the high-frequency component, rather than the low-frequency component(s), of an impedance spectrum, which characterizes the electrical properties of a PSC. In particular, it is shown that analysis of the high-frequency peak, only, can return the same value of the so-called ideality factor as the Suns- $V_{\mathrm{OC}}$ method. The agreement between these values indicates that such results should also be interpreted in terms of the ectypal diode theory.

\section{CONCLUSIONS}

In response to reports of unusual and unexplained values of the classical diode ideality factor for metal halide perovskite solar cells, we propose an alternative analytic model that is tailored to the unique properties of a planar PSC. We term this the ectypal diode theory for steadystate operation. Like the classical theory, this theory is derived as an approximation to a charge-transport model that describes the drift and diffusion of mobile charges across a device. While the charge transport model for a typical $p-n$ or $p-i-n$ type solar cell need only describe the motion of electrons and holes, the charge-transport model for a PSC must incorporate an additional drift-diffusion equation for the migration of mobile ionic charge that exists within the perovskite layer of a PSC. According to our systematic approximation of such a model, the presence of mobile ionic charge in the perovskite layer leads to an analog of the classical diode equation in which the ideality factor is replaced by a voltage-dependent value, which we term the ectypal factor. By comparison to numerical simulations, we verify that the ectypal diode equation is able to accurately reproduce the $J-V$ characteristics of efficient PSCs measured at sufficiently slow scan rates.

We explain, using two example sets of cell parameters and by considering five different forms of recombination, how the value of the ectypal factor can be used to interpret the steady-state $J-V$ characteristics of planar PSCs, using the same standard techniques that are used to obtain values of the ideality factor. Such techniques include the dark $J-V$ method and the Suns- $V_{\mathrm{OC}}$ method. In particular, the value of the ectypal factor depends on the form of the dominant recombination mechanism in a PSC as well as the distribution of the electric potential across the device. We then discuss the implications of the ectypal diode theory on experimental results presented in the literature. An important point to take from this work is that it is necessary to understand the distribution of the electric potential across a particular cell before drawing any conclusions about the type and location of its performance-limiting recombination mechanism, using standard techniques. However, by using the ectypal diode theory, these techniques can still be used to identify targets for the future development of PSCs.

Further mathematical analysis is required to determine the extent of the validity of the ectypal diode theory. In particular, future studies should analyze the solution to the full set of equations at high and low voltages as well as higher illumination intensities. The importance of this work lies in the realization that so-called "ideality factors" for PSCs have a different physical origin to other types of solar cell due to the presence of mobile ions. As a result, the classical interpretation can no longer be used to interpret the results of standard measurement techniques. However, the ectypal factor is a meaningful replacement that is intrinsically voltage dependent and captures the dependence of the dominant recombination mechanism on the electric field across a PSC. As such, it is proposed that the ectypal factor should be used in place of the ideality factor in the field of PSCs as a first step in analyzing their complex behavior.

By fitting steady-state experimental data to the ectypal diode equation, the values of physical parameters can be estimated and used as inputs to dynamic numerical 
simulations, which should enable researchers to achieve improved accuracy between theory and experiment for transient $J-V$ data. Such numerical simulations can be carried out using our open-source PSC simulation tool [43]. Alongside detailed numerical modeling, the ectypal diode equation offers researchers in the field of PSCs a valuable and practical tool with which to quantify the effects of ionic accumulation and charge-carrier recombination on the steady-state performance of PSCs.

\section{ACKNOWLEDGMENTS}

N.E.C. thanks Dr Giles Richardson, Professor Juan A. Anta and Laurence Bennett for their useful comments on the manuscript. N.E.C. is supported by an EPSRC Doctoral Prize (ref. EP/R513325/1).

\section{APPENDIX A: DERIVATION OF THE ECTYPAL DIODE EQUATION}

\section{Charge-transport model}

A complete mathematical model for ion migration and charge transport across the three core layers of a PSC is given in the work accompanying the release of the opensource simulation tool IonMonger [43]. The three core layers of a PSC are the electron transport layer, the perovskite absorber layer and the hole transport layer. These three layers are sandwiched between two metal contacts. The transport layers are assumed to be highly selective due to intentional doping or a high effective doping density. The model is specified by Eqs. (1)-(22) in Secs. 3.1-3.2 of that work and comprises of a full set of drift-diffusion equations for the ion vacancy, electron and hole densities, coupled to Poisson's equation for the electric potential. A very similar model is also given in our previous work [33] by Eqs. (1)-(15). The only difference is that the model in Ref. [33] uses a couple of extra approximations on the properties of the TLs, reducing the number of unknown physical parameters. The model in Ref. [43] forms the basis for the analysis presented in this work.

Richardson and coworkers [32,37] previously considered a single-layer model in which the two TLs are approximated as ideal metallic contacts and so the problem is reduced to a model that explicitly describes only the PAL. In Ref. [37], it is shown that the charge-carrier concentrations have a negligible effect on the electric potential, in comparison to the ion vacancy distribution. The accumulation of ionic charge adjacent to each of the PAL-TL interfaces leads to sharp changes in potential across narrow Debye layers. According to the principle of conservation, the ionic charge density stored in each perovskite Debye layer must be equal and opposite. As a result, the behavior of the cell depends on the evolution of the ionic accumulation and the corresponding potential drops across the Debye layers.
The asymptotic simplification carried out in Ref. [37] is extended to the full three-layer model for a PSC in Ref. [33]. It is found that the potential profile is predominantly determined by the ionic distribution within the PAL in combination with a couple of key properties of the TLs, namely the permittivity and the effective doping density. The effects of these TL properties are captured in the definitions of two dimensionless parameters, which are defined as

$$
\Omega_{E}=\sqrt{\frac{\varepsilon_{A} N_{0}}{\varepsilon_{E} d_{E}}}, \quad \text { and } \quad \Omega_{H}=\sqrt{\frac{\varepsilon_{A} N_{0}}{\varepsilon_{H} d_{H}}}
$$

where $\varepsilon_{E, A, H}$ are the permittivities of the ETL, PAL, and HTL, respectively, $N_{0}$ is the mean density of ion vacancies in the PAL, and $d_{E, H}$ are the effective doping densities of the ETL and HTL, respectively. The effects of varying these parameters are investigated in Ref. [33].

The dominant effect of the ionic distribution means that, as in the single-layer model, the behavior of the cell is controlled by the evolution of the ionic charge in the Debye layers. As a result, the model is reduced to the surfacepolarization model for planar PSCs comprising of a single ordinary differential equation (ODE) for the evolution of ionic charge in the Debye layers coupled to a steady-state boundary value problem (BVP) for the charge-carrier concentrations at a given time. The ODE for the evolution of ionic charge stored in the Debye layer on the righthand side of the perovskite $[Q(t)]$ is described by Eqs. (19)-(22) in Ref. [33]. These four equations are restated in Eqs. (A2)-(A5) using the notation in Tables III and IV.

The evolution of the charge density is governed by

$$
\frac{d Q}{d t}=\frac{q D_{I} N_{0}}{V_{T}} E_{\mathrm{bulk}}(t)
$$

where $E_{\text {bulk }}(t)$ is the bulk electric field, given by

$$
E_{\text {bulk }}(t)=\frac{1}{b}\left(V_{\mathrm{bi}}-V-V_{1}-V_{2}-V_{3}-V_{4}\right),
$$

in which $V_{\text {bi }}$ is the cell's built-in voltage, $V$ is the applied voltage, and $V_{1-4}$ are the potential drops across the Debye layers illustrated in Fig. 2, which obey the following

TABLE III. List of the model variables used to describe the internal state of the bulk of the PAL in the steady-state surfacepolarization model.

\begin{tabular}{ll}
\hline \hline Symbol & Description \\
\hline$p(x)$ & Bulk hole concentration \\
$j^{p}(x)$ & Hole current density \\
$n(x)$ & Bulk electron concentration \\
$j^{n}(x)$ & Electron current density \\
\hline \hline
\end{tabular}


TABLE IV. List of parameters for the steady-state surfacepolarization model.

\begin{tabular}{ll}
\hline \hline Symbol & Description \\
\hline$q$ & Elementary charge \\
$V_{T}$ & Thermal voltage \\
$D_{p}$ & Hole diffusion coefficient \\
$D_{n}$ & Electron diffusion coefficient \\
$\varepsilon_{A}$ & Permittivity of perovskite \\
$E_{\text {bulk }}$ & Electric field in the bulk of the PAL \\
$G(x)$ & Charge-carrier generation rate \\
$R(n, p)$ & Charge-carrier recombination rate \\
\hline \hline
\end{tabular}

capacitance relations:

$$
\begin{aligned}
& V_{1}=-\mathcal{V}\left[-\Omega_{E} Q(t)\right], \quad V_{2}=-\mathcal{V}[-Q(t)], \\
& V_{3}=\mathcal{V}[Q(t)], \quad V_{4}=-\mathcal{V}\left[-\Omega_{H} Q(t)\right] .
\end{aligned}
$$

Here, the function $\mathcal{V}(Q)$ is the inverse of the function $Q(\mathcal{V})$, defined by

$$
Q(\mathcal{V})=q N_{0} L_{D} \operatorname{sign}(\mathcal{V}) \sqrt{2}\left(e^{\mathcal{V} / V_{T}}-\mathcal{V} / V_{T}-1\right)^{1 / 2}
$$

in which $L_{D}$ is the perovskite Debye length defined by $\sqrt{\varepsilon_{A} V_{T} /\left(q N_{0}\right)}$.

From the solution of Eqs. (A2)-(A5), the electric field in the bulk of the PAL can be calculated from Eq. (A3). For a steady-state problem, $E_{\text {bulk }} \equiv 0$ and therefore $V_{1-4}$ satisfy

$$
V_{\mathrm{bi}}-V=V_{1}+V_{2}+V_{3}+V_{4}
$$

Therefore, $V_{1-4}$ can be considered to be functions of the applied voltage, which can be evaluated by numerical solution of Eqs. (A4)-(A6) for $Q$ and then substituting $Q$ back into Eq. (A4).

When the values of $E_{\text {bulk }}$ and $V_{1-4}$ are known, the charge-carrier concentrations ( $n$ and $p$ ) can be calculated from Eqs. (3)-(6) stated in the Supplemental Material of Ref. [33]. To obtain the $J-V$ characteristics of a PSC, the final step is to calculate the current density from Eq. (7) in the Supplemental Material of Ref. [33]. These equations are restated below with the steady-state assumption that $E_{\text {bulk }}=0$ and that $V_{1-4}$ are known functions of the applied voltage.

\section{Steady-state surface-polarization model}

The steady-state surface-polarization model for the charge-carrier concentrations in the PAL of a PSC consists of conservation equations for the electrons and holes within the bulk of the PAL $(0<x<b$, excluding the narrow Debye layers). The distribution of the electric potential between the four Debye layers is assumed to be known and governed by the distribution of mobile ionic charge. Using the notation in Tables III and IV, the conservation equations are

$$
-\frac{1}{q} \frac{\partial j^{n}}{\partial x}=G(x)-R(n, p), \quad j^{n}=q D_{n} \frac{\partial n}{\partial x}
$$

TABLE V. Table showing the analytic expressions that can be substituted into Eq. (A26) to give the approximate dark current density for each of the five different recombination rates considered in this work. Note that each of the potential drops $V_{1-4}$ is a function of the applied voltage $V$, while all other symbols are constants that are defined within the Supplemental Material [49]. The ectypal factor, introduced in this work, is defined by $\left(V_{\mathrm{bi}}-V\right) / F(V)$, see Table I.

\begin{tabular}{lccc}
\hline \hline Type of recombination & Form & $J_{d}$ & $F(V)$ \\
\hline Bimolecular bulk recombination & $R \sim \beta n p$ & $\frac{q b \beta d_{H} d_{E} g_{v} g_{c}}{g_{v}^{H} g_{c}^{E}} \exp \left(\frac{E_{v}-E_{v}^{H}-E_{c}+E_{c}^{E}}{q V_{T}}\right)$ & $V_{1}+V_{2}+V_{3}+V_{4}$ \\
Hole-limited bulk SRH & $R \sim \frac{p}{\tau_{p}}$ & $=q b \beta n_{i}^{2} \exp \left(\frac{V_{\mathrm{bi}}}{V_{T}}\right)$ & $V_{3}+V_{4}$ \\
Electron-limited bulk SRH & $R \sim \frac{n}{\tau_{n}}$ & $\frac{q b d_{H} g_{v}}{\tau_{p} g_{v}^{H}} \exp \left(\frac{E_{v}-E_{v}^{H}}{q V_{T}}\right)$ & $V_{1}+V_{2}$ \\
ETL-PAL interface SRH & $R_{l} \sim v_{p, E} p^{+}$ & $\frac{q b d_{E} g_{c}}{\tau_{n} g_{c}^{E}} \exp \left(-\frac{E_{c}-E_{c}^{E}}{q V_{T}}\right)$ & $V_{2}+V_{3}+V_{4}$ \\
PAL-HTL interface SRH & $R_{r} \sim v_{n, H} n^{-}$ & $\frac{q v_{n, H} d_{E} g_{c}}{g_{c}^{E}} \exp \left(-\frac{E_{c}-E_{c}^{E}}{q V_{T}}\right)$ & $V_{1}+V_{2}+V_{3}$ \\
\hline \hline
\end{tabular}


The accompanying boundary conditions (on the bulkfacing edge of each perovskite Debye layer) are

$$
\begin{aligned}
& n=n_{b} \exp \left(-\frac{V_{1}+V_{2}}{V_{T}}\right), \quad j^{p}=-q R_{l}, \quad \text { at } x=0^{+}, \\
& p=p_{b} \exp \left(-\frac{V_{3}+V_{4}}{V_{T}}\right), \quad j^{n}=-q R_{r}, \quad \text { at } x=b^{-},
\end{aligned}
$$

where the constants $n_{b}$ and $p_{b}$ take into account the discontinuity in the relevant carrier concentration across each interface, according to Boltzmann statistics for nondegenerate semiconductors, and are given by

$$
\begin{aligned}
& n_{b}=\frac{d_{E} g_{c}}{g_{c}^{E}} \exp \left(\frac{E_{c}^{E}-E_{c}}{V_{T}}\right), \\
& p_{b}=\frac{d_{H} g_{v}}{g_{v}^{H}} \exp \left(\frac{E_{v}-E_{v}^{H}}{V_{T}}\right) .
\end{aligned}
$$

Since carrier concentrations are discontinuous across an interface between two different materials, the interfacial recombination rates $R_{l}$ and $R_{r}$ are defined as functions of the electron concentration on the left-hand side of the relevant interface and the hole concentration on the right, i.e.,

$$
\begin{aligned}
& R_{l}(n, p)=R_{l}\left[d_{E} \exp \left(-\frac{V_{1}}{V_{T}}\right),\left.p\right|_{x=0^{+}} \exp \left(-\frac{V_{2}}{V_{T}}\right)\right], \\
& R_{r}(n, p)=R_{r}\left[\left.n\right|_{x=b^{-}} \exp \left(-\frac{V_{3}}{V_{T}}\right), d_{H} \exp \left(-\frac{V_{4}}{V_{T}}\right)\right] .
\end{aligned}
$$

Note that, in the boundary conditions and the expressions above, the exponential factors account for the rapid changes in the carrier concentrations across each Debye layer.

The current density $J$ generated by the PSC can be obtained from a solution for $n(x, t)$ and $p(x, t)$ for the PDE problem Eqs. (A7)-(A14) as follows. By integrating the difference between Eqs. (A7a) and (A8a), we find that the total current density $\left(J=j^{n}+j^{p}\right)$ is uniform across the perovskite layer. Then, by integrating the sum of Eqs. (A7a) and (A8a) over the width of the perovskite layer and applying the boundary conditions (A10b) and (A10b), we find that the current density is given by

$$
J=\int_{0}^{b} q[G(x)-R(n, p)] d x-q R_{l}-q R_{r} .
$$

Note that, in order for an analytic expression for the current density to be found, it is necessary to simplify the nonlinear PDE problem.

In both the numerical simulations and the following analysis, we use a simplified version of the Beer-Lambert law of light absorption, i.e., we assume that

$$
G(x)=I_{s} F_{\mathrm{ph}} \alpha \exp (-\alpha x),
$$

in which $F_{\mathrm{ph}}$ denotes the flux of photons incident on the light-facing perovskite surface (after accounting for reflection) under the equivalent of 1-Sun illumination; $\alpha$ is the light absorption coefficient of the perovskite; and, $I_{s}$ is the intensity of the illumination in Sun equivalents. Hence,

$$
J=J_{s}-J_{\text {dark }}
$$

where

$$
\begin{aligned}
J_{s} & =\int_{0}^{b} q G(x) d x=q I_{s} F_{\mathrm{ph}}[1-\exp (-\alpha b)], \\
J_{\text {dark }} & =\int_{0}^{b} q R(n, p) d x+q R_{l}+q R_{r} .
\end{aligned}
$$

\section{Simplifying assumptions}

Here, a set of three physically justifiable assumptions are applied to the BVP for the charge-carrier concentrations comprising of Eqs. (A7)-(A14). The simplified BVP is then solved for five different recombination rates. The result of this analysis is an expression for the steady-state current density $J$, which is written simply in terms of the four Debye layer potential drops $V_{1-4}$ indicated in Fig. 2 .

We make the following assumptions for a PSC operating at applied voltages within its power-generating regime $\left(0<V<V_{\mathrm{OC}}\right.$, as measured under the equivalent of 1-Sun illumination).

1. The rate of charge-carrier diffusion towards the PAL-TL interfaces dominates over the rate of chargecarrier recombination via interfacial trap states, for both holes and electrons within the PAL, i.e.,

$$
\begin{aligned}
& v_{p, E} \exp \left(-\frac{V_{2}}{V_{T}}\right) \ll \frac{D_{p}}{b}, \\
& v_{n, H} \exp \left(-\frac{V_{3}}{V_{T}}\right) \ll \frac{D_{n}}{b} .
\end{aligned}
$$


2. The perovskite layer width $(b)$ is much shorter than the carrier diffusion lengths, meaning that the rate of diffusion across the PAL is significantly greater than the rate of recombination within the bulk of the PAL, i.e.,

$$
\begin{aligned}
b & \ll \sqrt{\frac{D_{p}}{\beta n_{b}}} \exp \left(\frac{V_{1}+V_{2}}{2 V_{T}}\right), \\
b & \ll \sqrt{\frac{D_{n}}{\beta p_{b}}} \exp \left(\frac{V_{3}+V_{4}}{2 V_{T}}\right), \\
b & \ll \begin{cases}\sqrt{\tau_{p} D_{p}} & \text { for hole-limited bulk SRH, or } \\
\sqrt{\tau_{n} D_{n}} & \text { for electron-limited bulk SRH. }\end{cases}
\end{aligned}
$$

3. The cell is held either in the dark or under only a moderate illumination intensity (up to around the equivalent of 1 Sun), such that the carrier flux densities due to photogeneration are less than those due to diffusion across the PAL, i.e.,

$$
\begin{aligned}
I_{s} F_{\mathrm{ph}} & \ll \frac{D_{p} p_{b}}{b} \exp \left(-\frac{V_{3}+V_{4}}{V_{T}}\right), \\
I_{S} F_{\mathrm{ph}} & \ll \frac{D_{n} n_{b}}{b} \exp \left(-\frac{V_{1}+V_{2}}{V_{T}}\right) .
\end{aligned}
$$

The outcome of these three assumptions is that the rates of recombination $\left(R, R_{l}, R_{r}\right)$ and generation $(G)$ have a negligible effect on the distribution of the carrier concentrations ( $n$ and $p$ ), in the range of voltages relevant to solar-cell operation. Note that assumptions 1 and 2 place an upper bound on the voltage, while assumption 3 imposes a lower bound. In other words, we assume that the diffusion of charge carriers across the cell is the dominant process for determining the charge-carrier distributions within the PAL and hence the rates of recombination and the total current density generated during steady-state operation of the cell. Perovskite materials are known for their long diffusion lengths [50-52] and, therefore, we believe that this is a reasonable assumption for any high-performing planar PSC. The approximations are consistent with parameter estimates for PSCs with low bulk recombination previously used in Ref. [33]. It should be noted that taking these limits does not eliminate any type of recombination from taking place within the cell. However, such assumptions will not be valid for inefficient cells with poor quality perovskite films and/or transport layers (particularly those with low carrier mobilities).

Applying the simplifying assumptions reduces the BVP (A7)-(A14) to the following equations:

$$
\begin{gathered}
\frac{\partial j^{n}}{\partial x}=0, \quad j^{n}=q D_{n} \frac{\partial n}{\partial x}, \\
\frac{\partial j^{p}}{\partial x}=0, \quad j^{p}=-q D_{p} \frac{\partial p}{\partial x},
\end{gathered}
$$

with the boundary conditions

$$
\begin{aligned}
& n=n_{b} \exp \left(-\frac{V_{1}+V_{2}}{V_{T}}\right), \quad j^{p}=0, \quad \text { at } x=0^{+}, \\
& p=p_{b} \exp \left(-\frac{V_{3}+V_{4}}{V_{T}}\right), \quad j^{n}=0, \quad \text { at } x=b^{-} .
\end{aligned}
$$

By integrating and applying the boundary conditions, we find that the carrier concentrations are approximately uniform across the bulk of the PAL and given by

$$
\left.\begin{array}{l}
n=n_{b} \exp \left(-\frac{V_{1}+V_{2}}{V_{T}}\right) \\
p=p_{b} \exp \left(-\frac{V_{3}+V_{4}}{V_{T}}\right)
\end{array}\right\} \text { for } 0<x<b .
$$

It is therefore possible to approximate the dark current density for each of the different types of recombination, using these estimates for $n$ and $p$.

In this work, we further assume that a typical cell is limited predominantly by a single type of recombination which can be well approximated by a monomolecular or bimolecular rate, i.e., that a cell is limited by one of the five rates listed in Table I. This assumption is consistent with our focus on modeling the power-generating quadrant of the current-voltage characteristics of a PSC where the product of the carrier concentrations satisfies $n p>n_{i}^{2}$. Note that the intrinsic carrier concentration is defined by the relation:

$$
n_{i}^{2}=g_{c} g_{v} \exp \left(-\frac{E_{g}}{k_{B} T}\right),
$$

where $E_{g}=E_{c}-E_{v}$ is the band gap of the perovskite.

According to Eqs. (A13)-(A14), the interface recombination rates must take into account the exponential change in concentration due to the potential drop across the Debye layer adjacent to the relevant PAL-TL interface in order to be written in terms of the charge-carrier concentrations ( $n$ and $p$ ) within the bulk of the PAL. Therefore, the interface recombination rates in Table I are approximated by

$$
\begin{aligned}
& R_{l}=v_{p, E} p \exp \left(-\frac{V_{2}}{V_{T}}\right), \\
& R_{r}=v_{n, H} n \exp \left(-\frac{V_{3}}{V_{T}}\right) .
\end{aligned}
$$

The result of these assumptions is that the dark current density (valid for a power-generating PSC) can be approximated and written in the following form for all five rates 
of recombination:

$$
J_{\text {dark }}=J_{d} \exp \left(-\frac{F(V)}{V_{T}}\right) .
$$

The definitions of $J_{d}$ (which has the units of a current density) and the function $F(V)$ corresponding to each of the five recombination mechanisms are listed in Table V. These definitions provide a vital link between physical properties of the three core layers of a PSC and its overall performance as a solar cell during steady-state operation. By substituting Eq. (A26) into Eq. (A17), we derive the ectypal diode equation for steady-state operation stated in Eq. (5).

Whilst it may seem that the model has been oversimplified, it can be seen from Sec. IV that the steady-state $J-V$ behavior predicted by the ectypal diode equation is in good agreement with the results of numerical simulations of the full charge-transport model. Crucially, the model is able to capture the exponential effects of the four characteristic potential drops $V_{1-4}$ on the rates of recombination associated with different recombination pathways. It is also notable that the ectypal diode equation takes the same form as that of the approximate Shockley diode equation stated in Eq. (2), in that $J_{s}$ is independent of the applied voltage, while $J_{\text {dark }}$ is independent of the photogeneration parameters and constitutes the current density in the dark due to recombination for $V \gg V_{T}$.

\section{Evaluating the ectypal diode equation}

In order to obtain results from the ectypal diode equation, the potential drops $V_{1-4}$ must first be calculated, as functions of the applied voltage, from Eqs. (A4)-(A6). For the chosen charge-transport model (which includes a single, positively charged species of mobile ionic charge), these equations form an implicit equation for the Debye layer charge density $Q(t)$, which can be solved using, for example, MATLAB's root-finding algorithm fsolve [54]. Once $V_{1-4}$ are determined, the current density can then be calculated from Eq. (5) using the relevant expressions for $J_{d}$ and $F(V)$ for the chosen recombination mechanism from Table V. Hence, the proposed model reduces the task of simulating a steady-state $J-V$ curve of a PSC to finding the numerical solution to a single implicit equation for the charge density $Q(t)$, followed by substitution into the ectypal diode equation [Eq. (5)] for steady-state operation:

$$
J(V)=J_{s}-J_{d} \exp \left(\frac{V-V_{\mathrm{bi}}}{n_{\mathrm{ec}} V_{T}}\right), \quad n_{\mathrm{ec}}=\frac{V_{\mathrm{bi}}-V}{F(V)} .
$$

\section{APPENDIX B: SERIES AND SHUNT RESISTANCE}

High fill factors are a sign of the promising potential of PSCs. However, in reality, the effects of series and shunt resistance (arising, for example, from contact resistances and short circuits caused by imperfections in the cell's construction) reduce the performance of a photovoltaic device below its theoretical performance, as investigated in this work. It is possible to incorporate external series and shunt resistance into the ectypal diode Eq. (5) in an analogous way to in the classical theory. The ectypal diode equation then becomes

$$
\begin{gathered}
J=J_{s}-J_{d} \exp \left(\frac{V-J A R_{s}-V_{\mathrm{bi}}}{n_{\mathrm{ec}} V_{T}}\right)-\frac{V-J A R_{s}}{R_{\mathrm{sh}}}, \\
n_{\mathrm{ec}}=\frac{V_{\mathrm{bi}}-\left(V-J A R_{s}\right)}{F\left(V-J A R_{s}\right)},
\end{gathered}
$$

in which $J\left(A \mathrm{~cm}^{-2}\right)$ is the photocurrent density, $A\left(\mathrm{~cm}^{2}\right)$ is the contact area of the solar cell, $R_{s}(\mathrm{Ohm})$ is the series resistance and $R_{\mathrm{sh}}(\mathrm{Ohm})$ is the shunt (or parallel) resistance. The equation for the distribution of the total potential difference across the cell, to replace equation (A6), is

$$
V_{\mathrm{bi}}-\left(V-J A R_{s}\right)=V_{1}+V_{2}+V_{3}+V_{4} .
$$

[1] J. Nelson, The Physics of Solar Cells (Imperial College Press, London, UK, 2003).

[2] A. Pockett, G. E. Eperon, T. A. Peltola, H. J. Snaith, A. B. Walker, L. M. Peter, and P. J. Cameron, Characterization of planar lead halide perovskite solar cells by impedance spectroscopy, open-circuit photovoltage decay, and intensity-modulated photovoltage/photocurrent spectroscopy, J. Phys. Chem. C 119, 3456 (2015).

[3] O. Almora, K. T. Cho, S. Aghazada, I. Zimmermann, G. J. Matt, C. J. Brabec, M. K. Nazeeruddin, and G. GarciaBelmonte, Discerning recombination mechanisms and ideality factors through impedance analysis of high-efficiency perovskite solar cells, Nano Energy 48, 63 (2018).

[4] P. Calado, D. Burkitt, J. Yao, J. Troughton, T. M. Watson, M. J. Carnie, A. M. Telford, B. C. O'Regan, J. Nelson, and P. R. F. Barnes, Identifying Dominant Recombination Mechanisms in Perovskite Solar Cells by Measuring the Transient Ideality Factor, Phys. Rev. Appl. 11, 044005 (2019).

[5] D. Kiermasch, A. Baumann, M. Fischer, V. Dyakonov, and $\mathrm{K}$. Tvingstedt, Revisiting lifetimes from transient electrical characterization of thin film solar cells; A capacitive concern evaluated for silicon, organic and perovskite devices, Energy Environ. Sci. 11, 629 (2018).

[6] W. Tress, M. Yavari, K. Domanski, P. Yadav, B. Niesen, J. P. C. Baena, A. Hagfeldt, and M. Graetzel, Interpretation and evolution of open-circuit voltage, recombination, ideality factor and subgap defect states during reversible light-soaking and irreversible degradation of perovskite solar cells, Energy Environ. Sci. 11, 151 (2018). 
[7] National Renewable Energy Laboratory (NREL) research cell record efficiency chart, http://www.nrel.gov/pv/assets/ images/efficiency-chart.png (2018).

[8] J.-P. Correa-Baena, A. Abate, M. Saliba, W. Tress, T. Jesper Jacobsson, M. Grätzel, and A. Hagfeldt, The rapid evolution of highly efficient perovskite solar cells, Energy Environ. Sci. 10, 710 (2017).

[9] M. Saliba, J. P. Correa-Baena, M. Grätzel, A. Hagfeldt, and A. Abate, Perovskite solar cells: From the atomic level to film quality and device performance, Angew. Chem. Int. Ed. 57, 2554 (2018).

[10] J. Werner, B. Niesen, and C. Ballif, Perovskite/silicon tandem solar cells: Marriage of convenience or true love story? - An overview, Adv. Mater. Interfaces 5, 1700731 (2018).

[11] A. J. Bett, P. S. C. Schulze, K. M. Winkler, Ö. S. Kabakli, I. Ketterer, L. E. Mundt, S. K. Reichmuth, G. Siefer, L. Cojocaru, L. Tutsch, M. Bivour, M. Hermle, S. W. Glunz, and J. C. Goldschmidt, Two-terminal perovskite silicon tandem solar cells with a high-bandgap perovskite absorber enabling voltages over 1.8 V, Prog. Photovoltaics: Res. Appl. 28, 99 (2020).

[12] J.-P. Correa-Baena, S.-H. Turren-Cruz, W. Tress, A. Hagfeldt, C. Aranda, L. Shooshtari, J. Bisquert, and A. Guerrero, Changes from bulk to surface recombination mechanisms between pristine and cycled perovskite solar cells, ACS Energy Lett. 2, 681 (2017).

[13] H. Tan, A. Jain, O. Voznyy, X. Lan, F. P. G. de Arquer, J. Z. Fan, R. Quintero-Bermudez, M. Yuan, B. Zhang, Y. Zhao, F. Fan, P. Li, L. N. Quan, Y. Zhao, Z.-H. Lu, Z. Yang, S. Hoogland, and E. H. Sargent, Efficient and stable solution-processed planar perovskite solar cells via contact passivation, Science 355, 722 (2017).

[14] M. Stolterfoht, C. M. Wolff, Y. Amir, A. Paulke, L. Perdigon-Toro, P. Caprioglio, and D. Neher, Approaching the fill factor Shockley Queisser limit in stable, dopant-free triple cation perovskite solar cells, Energy Environ. Sci. 10, 1530 (2017).

[15] L. Contreras-Bernal, M. Salado, A. Todinova, L. Calio, S. Ahmad, J. Idígoras, and J. A. Anta, Origin and whereabouts of recombination in perovskite solar cells, J. Phys. Chem. C 121, 9705 (2017).

[16] P. Caprioglio, M. Stolterfoht, C. M. Wolff, T. Unold, B. Rech, S. Albrecht, and D. Neher, On the relation between the open-circuit voltage and quasi-Fermi level splitting in efficient perovskite solar cells, Adv. Energy Mater. 9, 1901631 (2019).

[17] B. Dänekamp, N. Droseros, D. Tsokkou, V. Brehm, P. P. Boix, M. Sessolo, N. Banerji, and H. J. Bolink, Influence of hole transport material ionization energy on the performance of perovskite solar cells, J. Mater. Chem. C 7, 523 (2019).

[18] M. Ansari-Rad, Y. Abdi, and E. Arzi, Reaction order and ideality factor in dye-sensitized nanocrystalline solar cells: A theoretical investigation, J. Phys. Chem. C 116, 10867 (2012).

[19] J. P. Gonzalez-Vazquez, G. Oskam, and J. A. Anta, Origin of nonlinear recombination in dye-sensitized solar cells: Interplay between charge transport and charge transfer, J. Phys. Chem. C 116, 22687 (2012).
[20] O. J. Sandberg, A. Sundqvist, M. Nyman, and R. Österbacka, Relating Charge Transport, Contact Properties, and Recombination to Open-Circuit Voltage in Sandwich-Type Thin-Film Solar Cells, Phys. Rev. Appl. 5, 044005 (2016).

[21] S. Wheeler, F. Deledalle, N. Tokmoldin, T. Kirchartz, J. Nelson, and J. R. Durrant, Influence of Surface Recombination on Charge-Carrier Kinetics in Organic Bulk Heterojunction Solar Cells with Nickel Oxide Interlayers, Phys. Rev. Appl. 4, 024020 (2015).

[22] A. Sundqvist, O. J. Sandberg, M. Nyman, J. H. Smått, and R. Österbacka, Origin of the s-shaped JV curve and the light-soaking issue in inverted organic solar cells, Adv. Energy Mater. 6, 1 (2016).

[23] L. Contreras-Bernal, S. Ramos-Terrón, A. Riquelme, P. P. Boix, J. Idígoras, I. Mora-Seró, and J. A. Anta, Impedance analysis of perovskite solar cells: A case study, J. Mater. Chem. A 7, 12191 (2019).

[24] G. J. A. Wetzelaer, M. Scheepers, A. M. Sempere, C. Momblona, J. Ávila, and H. J. Bolink, Trap-assisted nonradiative recombination in organic-inorganic perovskite solar cells, Adv. Mater. 27, 1837 (2015).

[25] A. Sadollahkhani, P. Liu, V. Leandri, M. Safdari, W. Zhang, and J. M. Gardner, Energetic barriers to interfacial charge transfer and ion movement in perovskite solar cells, ChemPhysChem 18, 3047 (2017).

[26] H. J. Snaith, A. Abate, J. M. Ball, G. E. Eperon, T. Leijtens, N. K. Noel, S. D. Stranks, J. T.-W. Wang, K. Wojciechowski, and W. Zhang, Anomalous hysteresis in perovskite solar cells, J. Phys. Chem. Lett. 5, 1511 (2014).

[27] R. S. Sanchez, V. Gonzalez-Pedro, J.-W. Lee, N.-G. Park, Y. S. Kang, I. Mora-Sero, and J. Bisquert, Slow dynamic processes in lead halide perovskite solar cells. Characteristic times and hysteresis, J. Phys. Chem. Lett. 5, 2357 (2014).

[28] E. L. Unger, E. T. Hoke, C. D. Bailie, W. H. Nguyen, A. R. Bowring, T. Heumüller, M. G. Christoforo, and M. D. McGehee, Hysteresis and transient behavior in currentvoltage measurements of hybrid-perovskite absorber solar cells, Energy Environ. Sci. 7, 3690 (2014).

[29] W. Tress, N. Marinova, T. Moehl, S. M. Zakeeruddin, M. K. Nazeeruddin, and M. Grätzel, Understanding the rate-dependent $\mathrm{J}-\mathrm{V}$ hysteresis, slow time component, and aging in $\mathrm{CH}_{3} \mathrm{NH}_{3} \mathrm{PbI}_{3}$ perovskite solar cells: The role of a compensated electric field, Energy Environ. Sci. 8, 995 (2015).

[30] J. W. Lee, S. G. Kim, J. M. Yang, Y. Yang, and N. G. Park, Verification and mitigation of ion migration in perovskite solar cells, APL Mater. 7, 041111 (2019).

[31] A. K. Jena, A. Kulkarni, and T. Miyasaka, Halide perovskite photovoltaics: Background, status, and future prospects, Chem. Rev. 119, 3036 (2019).

[32] G. Richardson, S. E. J. O'Kane, R. G. Niemann, T. A. Peltola, J. M. Foster, P. J. Cameron, and A. B. Walker, Can slow-moving ions explain hysteresis in the current-voltage curves of perovskite solar cells? Energy Environ. Sci. 9, 1476 (2016).

[33] N. E. Courtier, J. M. Cave, J. M. Foster, A. B. Walker, and G. Richardson, How transport layer properties affect perovskite solar cell performance: Insights from a coupled charge transport/ion migration model, Energy Environ. Sci. 12, 396 (2019). 
[34] M. T. Neukom, A. Schiller, S. Züfle, E. Knapp, J. Ávila, D. P. del Rey, C. Dreessen, K. P. Zanoni, M. Sessolo, H. J. Bolink, and B. Ruhstaller, Consistent device simulation model describing perovskite solar cells in steady-state, transient and frequency domain, ACS Appl. Mater. Interfaces 11, 23320 (2019).

[35] D. Walter, A. Fell, Y. Wu, T. Duong, C. Barugkin, N. Wu, T. P. White, and K. J. Weber, Transient photovoltage in perovskite solar cells: Interaction of trap-mediated recombination and migration of multiple ionic species, J. Phys. Chem. C 122, 11270 (2018).

[36] A. Walsh, D. O. Scanlon, S. Chen, X. G. Gong, and S.-H. Wei, Self-regulation mechanism for charged point defects in hybrid halide perovskites, Angew. Chem. Int. Ed. 54, 1791 (2015).

[37] N. E. Courtier, J. M. Foster, S. E. J. O'Kane, A. B. Walker, and G. Richardson, Systematic derivation of a surface polarisation model for planar perovskite solar cells, Eur. J. Appl. Math. 30, 427 (2019).

[38] S. Weber, I. M. Hermes, S. H. Turren Cruz, C. Gort, V. W. Bergmann, L. Gilson, A. Hagfeldt, M. Grätzel, W. Tress, and R. Berger, How the formation of interfacial charge causes hysteresis in perovskite solar cells, Energy Environ. Sci. 11, 2404 (2018).

[39] I. M. Hermes, Y. Hou, V. W. Bergmann, C. J. Brabec, and S. A. L. Weber, The interplay of contact layers: How the electron transport layer influences interfacial recombination and hole extraction in perovskite solar cells, J. Phys. Chem. Lett. 9, 6249 (2018).

[40] M. Cai, N. Ishida, X. Li, X. Yang, T. Noda, Y. Wu, F. Xie, H. Naito, D. Fujita, and L. Han, Control of electrical potential distribution for high-performance perovskite solar cells, Joule 2, 296 (2018).

[41] K. Taretto, New explicit current/voltage equation for $p$ i-n solar cells including interface potential drops and drift/diffusion transport, Prog. Photovoltaics: Res. Appl. 22, 870 (2014).

[42] The word ectype means a copy or reproduction made in the image of the archetype or ideal version.

[43] N. E. Courtier, J. M. Cave, A. B. Walker, G. Richardson, and J. M. Foster, IonMonger: A free and fast planar perovskite solar cell simulator with coupled ion vacancy and charge carrier dynamics, J. Comput. Electron. 18, 1435 (2019).

[44] C. P. Please, An analysis of semiconductor PN junctions, IMA J. Appl. Math. 28, 301 (1982).

[45] M. J. Ward, L. G. Reyna, and F. M. Odeh, Multiple steadystate solutions in a multijunction semiconductor device, SIAM J. Appl. Math. 51, 90 (1991).
[46] T. Kirchartz, B. E. Pieters, J. Kirkpatrick, U. Rau, and J. Nelson, Recombination via tail states in polythiophene:fullerene solar cells, Phys. Rev. B 83, 115209 (2011).

[47] S. Ravishankar, O. Almora, C. Echeverría-Arrondo, E. Ghahremanirad, C. Aranda, A. Guerrero, F. FabregatSantiago, A. Zaban, G. Garcia-Belmonte, and J. Bisquert, Surface polarization model for the dynamic hysteresis of perovskite solar cells, J. Phys. Chem. Lett. 8, 915 (2017).

[48] D. A. Jacobs, H. Shen, F. Pfeffer, J. Peng, T. P. White, F. J. Beck, and K. R. Catchpole, The two faces of capacitance: New interpretations for electrical impedance measurements of perovskite solar cells and their relation to hysteresis, J. Appl. Phys. 124, 225702 (2018).

[49] See Supplemental Material at http://link.aps.org/supple mental/10.1103/PhysRevApplied.14.024031 for tables of the parameter values used in this work and an additional figure.

[50] S. D. Stranks, G. E. Eperon, G. Grancini, C. Menelaou, M. J. P. Alcocer, T. Leijtens, L. M. Herz, A. Petrozza, and H. J. Snaith, Electron-hole diffusion lengths exceeding 1 micrometer in an organometal trihalide perovskite absorber, Science 342, 341 (2013).

[51] Q. Dong, Y. Fang, Y. Shao, P. Mulligan, J. Qiu, L. Cao, and J. Huang, Electron-hole diffusion lengths $>175 \mu \mathrm{m}$ in solution-grown $\mathrm{CH}_{3} \mathrm{NH}_{3} \mathrm{PbI}_{3}$ single crystals, Science $\mathbf{3 4 7}$, 967 (2015).

[52] T. Kirchartz, High open-circuit voltages in lead-halide perovskite solar cells: Experiment, theory and open questions, Philos. Trans. R. Soc. A: Math., Phys. Eng. Sci. 377, 20180286 (2019).

[53] One might argue that photogenerated electrons only have to overcome the potential drop $V_{3}$ to recombine at the PAL-HTL interface, however, the probability of a minority carrier recombining depends on the competition between extraction and recombination and hence $F(V)$ must take into account the positive effect that $V_{1}+V_{2}$ has on charge extraction, transporting photogenerated electrons away from the PAL-HTL interface and preventing recombination. At higher light intensities, when photogenerated carriers can no longer be efficiently extracted from the PAL, the potential barrier for this type of recombination may reduce to $V_{3}$.

[54] MATLAB, MATLAB version 9.3.0.713579 (R2017b) (2017).

[55] N. E. Courtier, G. Richardson, and J. M. Foster, A fast and robust numerical scheme for solving models of charge carrier transport and ion vacancy motion in perovskite solar cells, Appl. Math. Model. 63, 329 (2018). 